\title{
Functional study of Leishmania braziliensis protein arginine methyltransferases (PRMTs) reveals that PRMT1 and PRMT5 are required for macrophage infection
}

Lucas Lorenzon ${ }^{1}$, José C. Quilles Junior ${ }^{1}$, Gustavo Daniel Campagnaro ${ }^{1}$, Leticia Almeida ${ }^{1}$, Flavio Protasio Veras ${ }^{2}$, Rubens D. M. Magalhães ${ }^{1}$, Juliana Alcoforado Diniz ${ }^{1}$, Tiago R. Ferreira ${ }^{3}$, Angela K. Cruz ${ }^{1}$

1 - Department of Cell and Molecular Biology, Ribeirão Preto Medical School, University of São Paulo, Ribeirão Preto, São Paulo, Brazil; 2 - Department of Pharmacology, Ribeirão Preto Medical School, University of São Paulo, Ribeirão Preto, São Paulo, Brazil; 3 - Laboratory of Parasitic Diseases, National Institutes of Allergy and Infectious Diseases, National Institutes of Health, Bethesda, MD, USA

\begin{abstract}
In trypanosomatids, regulation of gene expression occurs mainly at the posttranscriptional level, and RNA-binding proteins (RBPs) are key players in determining the fates of transcripts. RBPs are major targets of protein arginine methyltransferases (PRMTs), which posttranslationally regulate the RNA-binding capacity and other macromolecular interactions of RBPs by transferring methyl groups to protein arginine residues. Herein, we present the results of a study that functionally characterized the five predicted PRMTs in Leishmania braziliensis by gene knockout and endogenous protein HA tagging using CRISPR/Cas9 gene editing. We report that arginine methylation profiles vary among Leishmania species and that target protein methylation changes across different $L$. braziliensis life cycle stages, with higher PRMT expression in the promastigote stages than in the axenic amastigote stage. Knockout of some of the L. braziliensis PRMTs led to significant changes in global arginine methylation patterns without affecting promastigote axenic growth. Deletion of either PRMT1 or PRMT3 disrupted most type I PRMT activity, resulting in a global increase in monomethyl arginine (MMA) levels, which is mainly catalyzed by PRMT7. Putative targets and/or PRMT-interacting proteins were identified by coimmunoprecipitation using HA-tagged PRMTs, revealing a network of target RBPs and suggesting functional interactions between them and a relevant participation in epigenetic control of gene expression. Finally, we demonstrate that L. braziliensis PRMT1 and PRMT5 are required for efficient macrophage infection in vitro, and that in the absence of PRMT1 and PRMT5, axenic amastigote proliferation is impaired. The results indicate that arginine methylation is modulated across life cycle stages in L. braziliensis and show possible functional overlap and cooperation among the different PRMTs in targeting proteins. Overall, our data suggest important regulatory roles of these proteins throughout the L. braziliensis life cycle, showing that arginine methylation is important for parasite-host cell interactions.
\end{abstract}




\section{Introduction}

Posttranslational modifications (PTMs) are known to regulate protein function in multiple processes, including the coordination of regulatory gene expression networks. Protein arginine methylation is a common PTM known to affect RNA-binding protein (RBP) activity (1-3) in several eukaryotes, including early-branching protozoa. The enzymes responsible for catalyzing the transfer of a methyl group from S-adenosyl-methionine to the terminal nitrogen of a peptidyl

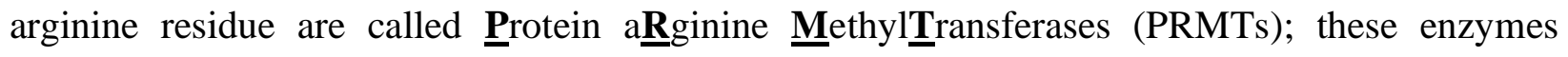
compose a nine-member family of proteins and have been identified in a large number of eukaryotes (4). PRMTs are involved in many cellular functions, from transcription regulation to RNA splicing, DNA repair, cell cycle and signal transduction (1). There is clear evidence that nucleic acid-binding proteins are the major substrates of PRMTs and that RBPs account for a large proportion of these PRMT substrates $(5,6)$. PRMTs are divided into three major types, all of which can catalyze $\omega-\mathrm{N}^{\mathrm{G}}$-monomethylarginine (MMA) as an initial methylation step; type I PRMTs catalyze the formation of asymmetric $\omega-\mathrm{N}^{\mathrm{G}}$-dimethylarginine (ADMA) as a final product, type II PRMTs produce symmetric $\omega-\mathrm{N}^{\mathrm{G}}$-dimethylarginine (SDMA) and type III PRMTs catalyze MMA alone. It is widely accepted that PRMT activity does not alter the charge of the target arginine residue, but an increase in hydrophobicity and mass may affect the hydrogen bonding capability of the target residue, thus positively or negatively modifying macromolecular interactions $(7,8)$.

Recent descriptions of PRMT (dys)function in many cellular mechanisms, including cancer progression and inflammatory responses, have emphasized the need for a better understanding of the role of arginine methylation in health and disease $(9,10)$. Notably, the importance of PRMTs in infectious diseases remains largely unknown; in particular, intracellular eukaryotic pathogens also express PRMT homologous genes, which may serve as putative drug targets for antiparasitic therapies.

Trypanosomatids, such as Trypanosoma spp. and Leishmania spp., are early-branching, medically relevant protozoa that carry five different classic PRMT genes (PRMT1, PRMT3, PRMT5, PRMT6 and PRMT7). In fact, these are the only protozoan parasites known thus far to 
harbor type I, II and III PRMTs, which suggests that arginine methylation may play a critical role during the life cycles of these protozoa $(11,12)$.

In addition to their medical relevance, trypanosomatids are unique eukaryotes because of their particular genetic organization that regulates gene expression, and this regulation of gene expression is mostly dependent on posttranscriptional regulatory mechanisms (13-16). Within the last two decades, thorough investigations of PRMTs and arginine methylation have been conducted in Trypanosoma brucei. Four active PRMT enzymes, namely, TbPRMT1ENZ, TbPRMT5, TbPRMT6, and TbPRMT7, and one prozyme (TbPRMT1 $\left.{ }^{\mathrm{PRO}}\right)$, previously known as TbPRMT3, have been characterized in T. brucei. Furthermore, fundamental knowledge about the enzymatic activities, effects of knockdown or knockout, functional interplay and substrate specificities of these enzymes has been obtained $(11,17-25)$.

Comparatively, much less is known about Leishmania PRMTs. Leishmania parasites are responsible for approximately 12 million cases of leishmaniasis worldwide; leishmaniasis is a human disease distributed in more than 80 countries in the tropical and subtropical regions of the globe that causes between 20 and 40 thousand deaths per year (26). To date, of the five classic PRMT homologs present in the Leishmania genome, only studies on Leishmania major PRMT7 have been reported (12). We have previously shown that the LmjPRMT7 levels are inversely correlated with the pathogenicity of $L$. major in vitro and in vivo $(27,28)$. In addition, Ferreira and coworkers showed that Leishmania PRMT7 acts as an epigenetic regulator of mRNA metabolism and provided mechanistic insight into the functional regulation of RBPs by methylation (29). Two of the identified LmjPRMT7 substrates, Alba3 and RBP16, were shown to be affected by this posttranslational modification in different ways. A global monomethyl arginine proteomic analysis revealed that approximately $3 \%$ of L. major proteins, including several RNA-binding proteins, have at least one methylated arginine residue, shedding light on the importance of this posttranslational modification for the parasite (29).

Given the relevance of improving the understanding of eukaryotic pathogens and PRMT function and the lack of information about Leishmania PRMTs, we present the first functional investigation of all five canonical PRMTs in Leishmania braziliensis, the main causative agent of tegumentary leishmaniasis in South America. We observed that although the three types of arginine methylation are observed in the three main biological forms of L. braziliensis, MMA is 
most likely the most common type. Immunoprecipitation assays revealed that R-methylated proteins are distributed in all cellular compartments, that RBPs are well represented among $L b$ PRMT targets and that some of the LbPRMTs may associate with each other in protein complexes. Moreover, our study reveals that the expression of both type I LbPRMT1 and type II LbPRMT5 is critical for establishing macrophage infection in vitro, suggesting that both SDMA and ADMA arginine methylation are important for the development of bona fide infectious $L$. braziliensis.

\section{Results}

\section{Conservation and divergence among PRMTs}

Global protein sequence alignments for each of the PRMTs were conducted, and these analyses were particularly focused on key motifs in the methyltransferase domain (6). We compared the protein sequences from L. braziliensis with their corresponding orthologs in T. brucei and humans. LbPRMT1 had $70.4 \%$ and $49 \%$ identity with TbPRMT1 ${ }^{\mathrm{ENZ}}$ and HsPRMT1, respectively; LbPRMT3 had 46.4\% and 24.2\% identity with these orthologs, respectively; LbPRMT5 had 29.4\% and $17.7 \%$ identity with these orthologs, respectively; LbPRMT6 had $46.6 \%$ and $24.9 \%$ identity with these orthologs, respectively; and LbPRMT7 had $50.7 \%$ and $21.5 \%$ identity with these orthologs, respectively. Overall, most of the six signature domains were conserved between the three species, but Motif III was particularly divergent when trypanosomatid PRMTs were compared with their human orthologs. Interestingly, both TbPRMT1 ${ }^{\mathrm{PRO}}$ and LbPRMT3 harbored mutations in the THW motif, but the Double E loop was altered only in TbPRMT1 ${ }^{\text {PRO }}$ (Fig. 1).

We also compared the sequences of PRMTs from parasitic and nonparasitic kinetoplastida. We observed that the motifs were highly conserved, with few exceptions. Interestingly, even the freeliving kinetoplastid Bodo saltans, the closest relative of representative trypanosomatid parasites, contained the same conserved signatures (Fig. S1), highlighting the conservation of these regions during evolution.

Differential arginine methylation patterns in distinct Leishmania species and parasite life cycle stages 
To evaluate arginine methylation in Leishmania, we used polyclonal antibodies that recognize each of the three major types of R-methylation (MMA, SDMA and ADMA) (Fig. 2a). As observed by western blotting, the three types of R-methylation were present in the promastigotes of different species of Leishmania (Fig. 2b, 2c and 2d), with evidence of slightly distinct R-methylation profiles, especially the MMA profiles, among the analyzed species. However, comparative quantitative analysis among the three different R-methylation types is not possible with this approach due to the differences in antibody origin and staining patterns used for each type. Next, we evaluated the profiles of R-methylated proteins in L. braziliensis throughout the progression of its life cycle, namely, in procyclic and metacyclic promastigotes and in axenic amastigotes (Fig. $\mathbf{2 e}, \mathbf{2 f}$ and $\mathbf{2 g}$ ). R-methylation was present in all the observed life cycle stages, with some detectable differences in the profiles, especially in the MMA and ADMA profiles, among these stages; most strikingly, a band of $\sim 33 \mathrm{kDa}$, containing ADMA, was enriched in the metacyclic promastigote stage compared to the other two stages. Moreover, as a general rule, amastigotes seemed to harbor a lower level of protein R-methylation than promastigotes (Fig. $\mathbf{2 f}$ and $\mathbf{2 h}$ ).

\section{PRMTs are mainly cytoplasmic proteins that are preferentially expressed in the promastigote stages}

The differences in the R-methylation profiles observed in L. braziliensis promastigotes and axenic amastigotes suggested that this PTM might be important for parasite development. Thus, we evaluated the expression profiles of the five putative $L b$ PRMTs in the three main stages of the life cycle. Due to the difficulties associated with genetically manipulating Leishmania spp. via classical homologous recombination techniques, we repurposed the CRISPR/Cas9 system previously developed by Beneke et al. (28) and generated an L. braziliensis strain expressing both Cas9 and T7 RNA polymerase, named pT007. The expression of Cas9 was confirmed by western blotting with antibodies against the Flag tag (Fig. 3a). Next, each of the PRMT coding sequences was endogenously tagged with three tandem copies of the HA tag at the 5 ' end (Fig. 3b). The correct insertion of the HA tag into both alleles of each targeted locus was confirmed by PCR (Fig. 3c); the transfectants were named tag1, tag3, tag5, tag6 and tag7 (where the number indicates each of the tagged PRMTs). The expression of the tagged PRMTs (HA-PRMT1, HA-PRMT3, HAPRMT5, HA-PRMT6 and HA-PRMT7) in the transfectants was confirmed by western blotting 
using protein extracts from procyclic promastigotes. The expected molecular weight of each PRMT was confirmed (Fig. 3d). The same L. braziliensis transfectants were used to evaluate the expression of the PRMTs across developmental stages (Fig. 3e). Western blotting results of protein extracts from procyclic and metacyclic promastigotes and axenic amastigotes indicated that the tagged PRMTs were preferentially expressed in promastigotes rather than in amastigotes (Fig. 3e). Decreased expression in amastigotes was observed for all the PRMTs, but this decrease in expression was particularly evident for PRMT7, which was barely detected in this biological form (Fig. 3e). We further investigated the subcellular distribution of the PRMTs using the same transfectants. Confocal microscopy analysis revealed differences in the intracellular distribution of the PRMTs: PRMT1, PRMT3 and PRMT5 were detected in both the cytoplasm and nucleus, PRMT6 was observed mainly in the intranuclear space, and PRMT7 was found only in the cytoplasm (Fig. 3f).

\section{Knockout of the PRMT1 and PRMT3 genes leads to changes in the arginine methylation profiles.}

To determine the biological relevance of the L. braziliensis PRMTs, we generated null mutants for each of their respective genes. Using established CRISPR/Cas9-expressing pT007 cells, we replaced each PRMT coding sequence with a blasticidin S resistance marker (BlastR, blasticidin deaminase; (30)) (Fig. 4a). Null mutants lacking each PRMT were generated by gene knockout (KO; $\Delta 1, \Delta 3, \Delta 5, \Delta 6$ and $\Delta 7$, where $\Delta n$ represents the deletion of the PRMTn gene), and the results demonstrated the nonessentiality of each individual PRMT for promastigote growth (Supplementary Fig. S2). However, the R-methylation profiles in some of these null mutants were significantly altered (Fig. $4 \mathbf{c}-\mathbf{e}$ ). Interestingly, both the $\Delta 1$ and $\Delta 3$ transfectants showed markedly increased levels of MMA and a less pronounced, but relevant, increase in the SDMA levels (Fig. $\mathbf{4 c}$ and $\mathbf{4 d}$, respectively). In contrast, in the absence of either PRMT1 or PRMT3, the levels of ADMA were drastically reduced (Fig. 4e). To validate these data, we generated constructs to add back each of the PRMTs to the corresponding KO parasites. HA-PRMTs, along with the neomycin phosphotransferase gene as a selectable marker, were inserted into the Small Subunit Ribosomal (SSU rDNA) locus (Fig. 4f). The integration of linearized DNA constructs into the target locus was confirmed (Supplementary Fig. S2), and the addback transfectants were named Add1, Add3, 
Add5, Add6 and Add7. The complementation of PRMT1 and PRMT3 clearly restored the MMA, SDMA and ADMA levels to those observed in the parental line (Fig. $\mathbf{4 g}, \mathbf{h}$ and $\mathbf{i}$ ).

\section{PRMT interactome analysis reveals multiple RNA-binding protein targets and suggests interplay between PRMTs}

The differential R-methylation profiles of the mutants lacking PRMT1 and PRMT3 led us to investigate the possible binding partners of each PRMT by coimmunoprecipitation (co-IP) assays. We used HA-tagged proteins to pull down the different PRMTs and the proteins with which they physically interact. The accurate immunoprecipitation of each PRMT was confirmed by Western blotting (Fig. 5a). A protein band of approximately $47 \mathrm{kDa}$ was detected in all the samples, and this molecular weight was consistent with the coeluted anti-HA antibody heavy chain and with the expected molecular weight of HA-PRMT3. The eluted protein complex was then analyzed by mass spectrometry to identify the proteins. After the removal of contaminating proteins (i.e., keratin, trypsin, serum albumin) and proteins identified in the WT sample (i.e., proteins not related to PRMT interactions), a total of 135 proteins were shown to interact with PRMTs. In summary, 77 proteins were pulled down with PRMT1, 38 with PRMT3, 66 with PRMT5, 44 with PRMT6 and 66 with PRMT7 (Table S1). Data analysis showed that most of the possible directly or indirectly interacting proteins were shared among PRMTs, while fewer proteins specifically interacted with only one PRMT (Fig. 5b). Among the 135 proteins identified, we searched for those with RG motifs, which are known to be preferential sites of R-methylation, and for proteins involved in RNA binding. We identified 20 proteins with one of these characteristics, such as several ATPdependent RNA helicases and proteins related to both RNA and DNA metabolism (NOL1/NOP2 rRNA methyltransferase, RNA cytidine acetyltransferase, and ruvb-like 1 DNA helicase, among others; Fig. 5c).

Interestingly, two other PRMTs were identified among PRMT1-interacting proteins: PRMT3, the binding protein with most unique peptides identified in this co-IP, and PRMT7 (Fig. 5d). Similarly, PRMT1 was the second most abundant PRMT3 binding partner (Fig. 5d). These findings suggest that L. braziliensis PRMTs could act as heterocomplexes or even functionally overlap, as already observed in other organisms, including $T$. brucei $(25,31)$. To investigate this hypothesis, we designed a strategy to knockout the three PRMT genes in the HA-tagged transfectants, aiming to 
determine whether the absence of either PRMT3 or PRMT7 could lead to changes in the PRMT1 expression levels (similar experiments were performed with the other possible combinations). These tag-knockout combinations were successfully generated (Fig. 5e). The results clearly showed that knockout of PRMT1 strongly reduced the expression of PRMT3, and the inverse analysis showed similar results, in both procyclic and metacyclic promastigotes (Fig. 5f). However, neither the knockout of PRMT1 nor PRMT3 altered the expression of PRMT7 (Fig. 5f).

These results, along with the nonessentiality of the PRMTs (Fig. 4b), prompted us to generate double knockout mutants and to evaluate the viability of the transfectants. Given the differential R-methylation profiles (Fig. 4c, 4d and 4e), we knocked out each of the other PRMTs on the PRMT1-null background, generating double null mutants (e.g., $\Delta 1 / 3$ to indicate the knockout of both the PRMT1 and PRMT3 genes). Surprisingly, all the double null transfectants we investigated were viable (Fig. 5g). We next asked whether the double knockout would also affect the differential R-methylation phenotype. Interestingly, the MMA and SDMA hypermethylation observed in $\Delta 1$ (Fig. 4c and 4d) was almost completely lost in the absence of PRMT7 (Fig. 5h and 5i).

\section{Monomethyl arginine IP reveals that diverse proteins are hypermethylated in PRMT1-null mutants}

Given the arginine methylation profiles observed in the $\Delta 1$ and $\Delta 3$ parasites (Fig. $4 \mathbf{c}$ and $4 \mathbf{d}$ ) together with the finding that PRMT7 is required for MMA accumulation, we aimed to identify proteins that were highly monomethylated in the $\Delta 1$ parasite. Using anti-MMA antibodies, we performed an IP experiment to identify MMA proteins in the pT007, $\Delta 1$, Add1 and $\Delta 1 / \Delta 7$ transfectants (Fig. 5j). Quantitative analysis of the mass spectrometry data indicated that 32 proteins were at least 2 times more abundant in $\Delta 1$ than in all the other transfectants. Among these proteins, RBPs, Rab proteins, one centromere-binding protein (cbf5), one RNA helicase and some uncharacterized proteins were noteworthy (Table 1), suggesting a role for L. braziliensis PRMTs in the control of nucleic acid-binding protein activities.

Using a complementary MS analysis approach on the same samples, we were also able to identify peptides bearing mono- or dimethylated arginine residues. Within the four samples (pT007, $\Delta 1$, Add1 and $\Delta 1 / \Delta 7$ ), we identified MMA peptides corresponding to 38 different proteins; of these proteins, 7 proteins harbored both mono- and dimethylated peptides (Table 2). Additionally, 14 
other proteins with DMA peptides were identified. Among these proteins, several putative RNA helicases, RBPs (including RBP16), rRNA methyltransferases and several uncharacterized proteins harboring more than one arginine methylated residue were noteworthy (Table 2 ).

\section{PRMT1- and PRMT5-null parasites fail to efficiently infect human and mouse macrophages in vitro}

To investigate the possible role of $L$. braziliensis PRMTs in parasite-host cell interactions, we first generated L. braziliensis parasites expressing both Cas9 and tdTomato (pT_Tomato, Fig. 6a and 6b); these parasites allowed the detection and quantitative analysis of in vitro infections in an automated manner (Fig. 6c). Next, we deleted each PRMT gene in the pT_Tomato parasites (Fig. 6d and 6e) and used fluorescently labeled null mutants for in vitro infection assays. Analyses of the infection profiles and intracellular survival rates were performed in three independent infection assays with the THP-1 monocyte-like cell line. Interestingly, the PRMT1- and PRMT5-null mutants infected both murine and human macrophages at lower ratios than the control parasites (in human macrophages $p<0.0005$ for both PRMT1 and PRMT5, in murine macrophages $p=0.08$ for PRMT1 and $p=0.0003$ for PRMT5). Both the percentage of infected THP-1 phagocytes and the number of intracellular amastigotes were significantly reduced $48 \mathrm{~h}$ postinfection with the two mutants (Fig. 6f and 6g). Strikingly, there were $\sim 50$-fold and $\sim 3$-fold decreases in the percentage of infected macrophages after infection with the $\Delta 1$ and $\Delta 5$ mutants, respectively, compared to the percentage of infected macrophages after infection with the control parasites (Fig. 6f). To validate these data, we generated addback parasites by returning the PRMT1 and PRMT5 genes to their own null mutants (Fig. 6h). The integration of the HA-tagged PRMT1 and PRMT5 genes to both alleles of their own loci was confirmed by multiplex PCR (Fig. 6h), and the expression of these proteins was observed by western blotting (Fig. 6i). Although a significant difference remained between the parental line and the $\Delta$ or addback lines, both the percentage of infected THP-1 cells and the number of amastigotes per infected cell were restored upon restoration of PRMT1 and PRMT5 expression (Fig. $6 \mathbf{f}$ and 6g). In addition, PRMT1, PRMT3 and PRMT5 KO parasites were submitted to a single infection assay with bone marrow-derived macrophages affecting similarly infection (BMDMs, Fig. S3). 
Given the differences observed between the parental and PRMT-KO lines regarding the intracellular survival of amastigotes, we sought to understand whether this was due to impaired resistance of $\Delta 1$ and $\Delta 5$ mutants to the antimicrobial macrophage machinery or due to impaired appropriate amastigogenesis or amastigote proliferation. We, thus, applied an axenic amastigogenesis strategy in which the differentiating parasites are not subjected to the phagosomal stress conditions (32), and measured the rate of parasite growth by MTT assay (Fig. 7a). Interestingly, although equivalent numbers of metacyclic promastigotes were purified from each PRMT-KO line and the parental pT_Tomato line (Fig. 7b), the differentiation of metacyclic promastigotes into amastigotes seemed to be impaired in the $\Delta 1, \Delta 3$ and $\Delta 5$ parasites, as suggested by an in vitro analysis (Fig. 7c). Concordantly, the number of amastigotes counted at the fourth day of in vitro differentiation were significantly reduced in these three mutants (Fig. 7c). The reexpression of each of these proteins in their respective null mutants restored their growth and survival to the same rates observed in the parental line (Fig 7c). Nevertheless, all the cell lines were able to survive being cultured at $33{ }^{\circ} \mathrm{C}$ for two passages in FBS (Fig. 7a) and could differentiate back into promastigotes (Supplementary Fig. S4).

\section{Discussion}

In this study, we characterized all the canonical protein arginine methyltransferases (PRMTs) in L. braziliensis. Among the five annotated L. braziliensis PRMTs (LbPRMTs), only LbPRMT3 lacks motifs characteristic of enzymatically active PRMTs. Notably, the global arginine methylation profiles of parasites are developmentally regulated as the parasite progresses from the procyclic promastigote stage to the metacyclic promastigote stage and the amastigote stage. This R-methylation profile also varies among the different Leishmania species evaluated. Most importantly, it is evident from this work and previous reports $(22,27,28)$ that parasite-derived arginine methylation plays a role in host-parasite interactions. Our data indicate that the expression of major type I and type II PRMTs (i.e., PRMT1 and PRMT5) is required for amastigote survival inside macrophages. The knockout of LbPRMT1 or LbPRMT5 inhibits axenic amastigote growth, which might partially explain the failure of these parasites to infect macrophages in vitro. Nevertheless, none of the PRMTs are essential for promastigote growth in axenic culture (Supplementary Fig. S5). 
The analysis of the conserved seFquence signatures in the methyltransferase domain revealed the presence of the previously identified key motifs for PRMT activity (6). The PRMT catalytic core is similar across all type I, II and III enzymes, and six signature motifs are relevant for methyltransferase function. Motif I contains three highly conserved glycines and forms the core of the AdoMet binding pocket; postmotif I and motif II are associated with noncovalent bonds and AdoMet binding pocket stabilization; key residues in the double-E motif position arginine residues in substrates; and finally, motif III forms a parallel $\beta$-sheet with motif II, and the THW loop is critical for substrate binding and for $\mathrm{N}$-terminal $\alpha$-helix stabilization. An extra motif, YFxxY, is conserved and located at the N-terminus of Type I PRMTs but absent from type II and III PRMTs (6).

Thus, the alignment of these motifs from L. braziliensis PRMTs with the thoroughly investigated motifs from T. brucei and Homo sapiens PRMTs highlighted the conservation of all six motifs. As expected, the conservation among the trypanosomatid sequences is higher, and it is notable that among the motifs, motif III is less conserved in the 5 sequences that were compared. In contrast to the other PRMTs, both $L b P R M T 3$ and TbPRMT1 ${ }^{\text {PRO }}$ harbor critical amino acid substitutions in the THW motif (Fig. 1). However, LbPRMT3 harbors two conserved glutamic acid residues that are essential for the function of the Double E loop motif, while TbPRMT1 ${ }^{\text {PRO }}$ does not.

Kafková et al. (2017) demonstrated that the enzyme previously named TbPRMT3 is not an active PRMT but acts as a TbPRMT1-like prozyme; therefore, it is currently named TbPRMT1 ${ }^{\text {PRO }}$. This protein forms a heterodimer with TbPRMT1 (currently known as TbPRMT1 ${ }^{\mathrm{ENZ}}$ ), and two heterodimers associate to form the active enzyme unit (21). Although LbPRMT3 possesses a conserved double E loop, the conservation of other sequence features between LbPRMT3 and TbPRMT1 ${ }^{\mathrm{PRO}}$ provides the first evidence that $L b$ PRMT3 might be, in fact, a prozyme; however, it is still too early to name this enzyme $L b \mathrm{PRMT} 1^{\mathrm{PRO}}$.

In addition, we aligned the five PRMTs from several trypanosomatids and a member of the Bodonidae family, Bodo saltans. The alignment results reveal clear subgroups of conservation levels and that the PRMTs from trypanosomes and Leishmania cluster together. Interestingly, the results also show that, despite divergences, the PRMT signature motifs are present in the enzymes 
of the nonparasitic earlier branching Bodo saltans species; one exception is the lack of the YFxxY motif in the PRMT6 ortholog, which may impair putative PRMT6 type I activity (Fig. S1).

Interestingly, $\Delta 1$ and $\Delta 3$ mutants displayed a marked reduction in the levels of ADMA, while displaying increased levels of SDMA, and a particularly marked increase of MMA levels (Fig. 4c-4e). These data indicate that, similar to T. brucei PRMT1 (25), L. braziliensis PRMT1 is the major type I PRMT expressed in the parasite. As PRMT1 activity was lost in $\Delta 1$ and $\Delta 3$ cells, the rate of consumption of MMA decreased, causing an accumulation of monomethylated arginine, which in turn increased the availability of monomethylated substrates for type II PRMT activity, leading to an elevation in SDMA levels. This is reinforced by the increased intracellular levels of ADMA, and consequent decrease in MMA and SDMA, upon the re-expression of PRMT1 and PRMT3 (Fig.4g-4i). The individual KO of any other PRMT showed subtle or imperceptible alterations in R-methylation profiles, including the KO of PRMT7, which is proposed to be the only type III PRMT expressed in trypanosomatids. Alternatively, PRMT7 might be responsible for a compensatory increase in activity when PRMT1 or PRMT3 expression is knocked out. Furthermore, we evaluated a group of combined PRMT knockout lines: knockout of two PRMT-encoding genes was performed to reveal the minimum set of classic PRMTs required for Leishmania survival. Interestingly, we observed that parasites with both PRMT1 and PRMT3, PRMT5, PRMT6 or PRMT7 knocked out were viable in axenic promastigote culture. Interestingly, $\Delta 1 / 7$ cells displayed a striking reduction in the levels of MMA, showing that, similar to what was observed in T. brucei (25), LbPRMT7 is the major catalyzer of MMA formation. The minor changes observed in the MMA profile of the $\Delta 7$ cells are likely due to the scavenging of monomethylation activities by other PRMTs. Furthermore, we could not observe any relevant alteration in the levels of arginine methylation upon the knockout of LbPRMT5, even though its ortholog in T. brucei (TbPRMT5) is the only type II PRMT characterized in that parasite, and it methylates a relatively broad range of substrates in vitro (18). These results may suggest that PRMTs are not at all essential for parasite survival in axenic culture, or these results may indicate redundancy between R-methylation writers and possible noncanonical PRMTs that have not yet been discovered in the Leishmania genome.

The expression levels of all the PRMTs vary throughout the Leishmania life cycle. PRMT expression is more abundant in promastigotes than in amastigotes, but in particular, the expression 
of PRMT5 and PRMT7 is lower in amastigotes (8\% and 1\% procyclic expression, respectively), and the latter is practically undetectable by western blotting (Fig. 3e). We have also shown that LbPRMTs are predominantly cytoplasmic, except for LbPRMT6, which is more abundant in the nucleus. LbPRMT1, LbPRMT3 and LbPRMT5 show dual nuclear-cytoplasmic localization. Considering that the major classes of arginine-methylated proteins are histones and RNA-binding proteins, it is expected that at least one of the PRMTs should be localized to the nuclear compartment. Since we only analyzed the subcellular localization of PRMTs in procyclic promastigotes, further investigation is necessary to draw conclusions about their localization in other stages of the life cycle.

Using the HA-tagged version of each L. braziliensis PRMT, immunoprecipitation assays were conducted to reveal possible targets of these proteins. A large number of proteins were pulled down (135), and we observed that $41 \%$ of the interacting proteins (55) were shared among the different PRMTs, leading us to speculate that the parasite has developed a certain redundancy in R-methylation; this redundancy might indicate the relevance of R-methylation for vital processes and at least partially explain why no individual PRMT is required for promastigote viability in vitro. Such overlap between PRMTs has been demonstrated in different organisms, including the close relative of L. braziliensis, T. brucei (25).

The IP assays also revealed that each PRMT has a variable group of unique interacting proteins (Table S1). In L. braziliensis, a large number of proteins were identified in the interactome, 12 of which were PRMT5-specific, 19 of which were PRMT1-specific, 6 of which were PRMT6 specific, and 16 of which were PRMT7 specific. Interestingly, PRMT3 IP pulled down only 2 unique proteins, supporting the hypothesis that PRMT3 does not have R-methylation activity on its own but possibly acts as a cofactor of PRMT1. These unique interacting proteins are of particular interest and should be further explored, especially those of LbPRMT1 and LbPRMT5, which are putative targets for antiparasitic chemotherapy, given their likely role in the parasitehost cell interaction. Importantly, our IP assays revealed an important number of RBPs, which points to the participation of L. braziliensis PRMTs as epigenetic regulators of gene expression control pathways in this parasite.

It is intriguing that even with a preferential expression profile in the insect forms, in vitro infection is negatively affected when $L b$ PRMT1 or LbPRMT5 are deleted, and our results indicate 
that LbPRMT1 expression is more abundant in amastigotes than LbPRMT5. It is unknown whether the levels of these proteins correlate with their activities in vivo; thus, even when present at very low or undetectable levels, these PRMTs might be able to methylate their targets in amastigotes. Therefore, the R-methylated proteins detected with anti-MMA and anti-SDMA or anti-ADMA antibodies (Fig. 2f, $2 \mathbf{g}$ and $\mathbf{2 h}$ ) could have been methylated in one of the previous developmental stages. Importantly, no arginine demethylase function has been reported in this parasite. In fact, we have previously observed that the PRMT7 levels in L. major markedly affect not only the rate of cell infection in vitro but also the outcome of murine infection in vivo, even though the LmjPRMT7 protein levels are largely decreased in the infectious stages of the life cycle $(27,28)$.

An interesting feature of $T$. brucei PRMT1 is its unique heterotetrameric nature; this complex is composed of two heterodimers containing an active PRMT, TbPRMT1 ${ }^{\mathrm{ENZ}}$, and a cofactor, $T b P R M T 1^{\mathrm{PRO}}$. Herein, we did not conduct any functional study to confirm or refute a similar organization of the PRMT1 complex. Nevertheless, we have indications that this is also the case in L. braziliensis. We observed that (i) both PRMT1- and PRMT3-knockout Leishmania mutants $(\Delta 1$ and $\Delta 3)$ exhibited similarly affected R-methylation profiles, that is, increased MMA and SDMA levels and decreased ADMA levels (Fig. 4c, 4d and 4e), (ii) $\Delta 1$ parasites had decreased levels of PRMT3 and vice versa (Fig. 5f); (iii) both proteins exhibited similar localization in procyclic promastigotes (Fig. 3d and 3f); (iv) only two PRMT3-specific interacting proteins were pulled down in the co-IP assays (Fig. 5b), and (v) PRMT3 lacked a conserved TWH motif.

Despite these features, knockout of the PRMT3 gene did not affect the infection profile in the in vitro infection assays, unlike knockout of the PRMT1 gene (Fig. 6f and 6g). A possible hypothesis would be that $L b$ PRMT1 may play a putative moonlight activity, unrelated to its Rmethylation activity, affecting macrophage infection. This hypothesis will be pursued further in future studies. Contrastingly, PRMT3-knockout parasites exhibited impaired axenic amastigote growth at levels similar to PRMT1 knockout parasites, which may be due to the absence of the host-derived factors that play a role in amastigote differentiation and proliferation in vivo.

Our data show that there is clear functional overlap among the different LbPRMTs and possible redundancy in their activities. Reverse genetic tools revealed that none of the PRMTs are essential for promastigote axenic growth, but further studies in sand flies would be an interesting future direction. This is supported by the finding that PRMT1- and PRMT5-knockout metacyclic 
promastigotes are impaired during infection in vitro and have reduced abilities to undergo amastigogenesis. In addition, we opened a new venue of investigation by reporting coimmunoprecipitated putative LbPRMT targets, particularly for PRMT1 and PRMT5. Several PRMT inhibitors are currently available for research, and some are already being assessed as anticancer therapies. Based on the phenotypic changes in L $b$ PRMT null mutants described here, inhibition of specific PRMT activity has the therapeutic potential to hinder parasite development in the infected mammalian host and should be tested as antiparasitic drug candidates. A chemical approach that targets Leishmania PRMTs may provide feasible ways to block key regulators of the parasite's gene expression and indirectly affect RNA metabolism.

\section{Methods}

Alignments. The protein sequences of the PRMTs from trypanosomatids and Bodo saltans were obtained from TriTrypDB (https://tritrypdb.org/tritrypdb/app), and the human PRMT sequences were obtained from NCBI (https://www.ncbi.nlm.nih.gov/). The sequences were aligned using Geneious v6.18 software (ClustalW - ID parameters). The key motifs of the conserved PRMT methyltransferase domain are shown. Colors indicate the conservation of residues: the darker the residues are, the more similar they are.

Parasite cultures. Promastigotes of Leishmania braziliensis strain M2903 (MHOM/BR/75/M2903) were cultured in M199 medium (Sigma-Aldrich) supplemented with $10 \%$ heat-inactivated fetal bovine serum as previously described (33). To obtain axenic amastigotes, $5 \times 10^{6}$ promastigotes in the stationary growth phase (fifth day) were inoculated into $10 \mathrm{~mL}$ of $100 \%$ fetal bovine serum and incubated at $33{ }^{\circ} \mathrm{C}$ in $5 \% \mathrm{CO}_{2}$ as previously described (34). Strains from other Leishmania species used were L. major LV39 (MRHO/SU/59/P), L. amazonensis PH8 (IFLA/BR/67/PH8) and L. infantum NCL (MHOM/BR/1972/LD).

Western blotting. Leishmania cells were pelleted $\left(7 \mathrm{~min}, 3{ }^{\circ} \mathrm{C}, 2000 \mathrm{xg}\right.$ ), washed with $500 \mu \mathrm{L}$ of ice-cold PBS containing 1X cOmplete protease inhibitors (Roche), resuspended in $\sim 10 \mu \mathrm{L}$ extraction buffer (SDS 2\%, $50 \mathrm{mM}$ Tris-Cl pH 7,4, $1 \mathrm{mM}$ PMSF, 1x cOmplete) per 1x10 ${ }^{6}$ cells and boiled for $10 \mathrm{~min}$. The protein concentrations of the samples were quantified by measuring the absorbance at $280 \mathrm{~nm}$ on a Nanodrop One instrument (Thermo Scientific), and the samples 
were mixed with $0.2 \mathrm{~V}$ of $6 \mathrm{X}$ sample buffer $(350 \mathrm{mM}$ Tris-Cl $\mathrm{pH} 6.8,30 \%$ glycerol, $10 \%$ SDS, $0.12 \mathrm{mg} / \mathrm{mL}$ bromophenol blue, 6\% 2-mercaptoethanol) and boiled for 3 more minutes. Approximately $40 \mu \mathrm{g}$ of protein extract was loaded per well in polyacrylamide gels. The proteins were transferred to nitrocellulose membranes (GE Healthcare Life Sciences - REF 10600003), which were incubated with the appropriate antibodies according to the manufacturer's instructions. Anti-MME (Cell Signaling Technologies [CST] - 8015S), anti-SME2 (CST - 13222), anti-AME2 (CST - 13522), anti-HA (Sigma - H3663), and anti-EF1 $\alpha$ (Merck - 05-235) were used for western blotting according to the manufacturer's instructions. Secondary anti-mouse and anti-rabbit peroxidase-conjugated antibodies (GE Healthcare - NA931V and NA934V, respectively) were used according to the manufacturer's instructions. The membranes were visualized using the chemiluminescent substrate of an ECL kit (GE Healthcare - RPN2232). Images were captured on ImageQuant LAS 4000 (GE Healthcare).

Single guide and donor DNA construction. The protocol for the construction of sgRNA templates was slightly modified from Beneke et al., 2017. For the amplification of sgRNA templates, $0.2 \mathrm{mM}$ dNTPs, $2 \mu \mathrm{M}$ each of primer G00 (sgRNA scaffold) and a gene-specific forward primer (primers 29-41 - Table S2), and 1 unit of Phusion DNA Polymerase (NEB) were mixed in $1 \times \mathrm{HF}$ buffer in a total volume of $50 \mu \mathrm{l}$. The PCR conditions were $30 \mathrm{~s}$ at $98{ }^{\circ} \mathrm{C}$ followed by 35 cycles of $10 \mathrm{~s}$ at $98^{\circ} \mathrm{C}, 30 \mathrm{~s}$ at $55^{\circ} \mathrm{C}$, and $15 \mathrm{~s}$ at $72^{\circ} \mathrm{C}$. A second PCR was carried out using $1 \mu 1$ of the abovementioned reaction as the template for amplification with G00_loredited_F and G00_loredited_R (same conditions; $50 \mu$ t total volume) to obtain higher yields. For donor DNA amplification, specific primers (primers $42-51$ and 14-18 in Table S2) were used to amplify the resistance genes from pTBlast or pTPuro (35) and 3xHA_Nterm_Blast (sequence 65 - Table S2). The PCR conditions were $30 \mathrm{~s}$ at $98^{\circ} \mathrm{C}$ followed by 35 cycles of $10 \mathrm{~s}$ at $98{ }^{\circ} \mathrm{C}, 40 \mathrm{~s}$ at $60{ }^{\circ} \mathrm{C}$, and $2 \mathrm{~min}$ at $72{ }^{\circ} \mathrm{C}$ ( 2 reactions of $50 \mu \mathrm{l}$ for each donor). All the reactions described above were combined and precipitated using ethanol and sodium acetate. The DNA pellet was resuspended in $60 \mu \mathrm{l}$ of $1 \mathrm{x}$ Tb-BSF buffer and used to transfect parasites in the log phase of growth. To generate the Addback transfectants (Fig. 4f-i), the PRMT1 and PRMT3 genes were amplified with primers 54-57 (Table S2) and cloned between the XhoI and BamHI sites of pSSU_neo_Tdtomato (36). The resultant plasmids were digested with PacI and PmeI (New England Biolabs) and used to transfect L. braziliensis $\Delta 1$ and $\Delta 3$. 
Transfections. Promastigotes in the log phase of growth were centrifuged at $2000 \mathrm{~g}$ at $25^{\circ} \mathrm{C}$ for 10 minutes. The pellets were resuspended in 1/10 V Tb-BSF buffer (37). The parasites were centrifuged under the same conditions and resuspended at a concentration of $2 \times 10^{8}$ parasites per $\mathrm{ml}$ in the same buffer. One hundred microliters of this suspension was mixed with the proper amount of DNA, and transfection was performed with an Amaxa Nucleofactor instrument (LONZA) using the X-001 program. The culture was maintained in M199 for 16 hours prior to further selection with the appropriate antibiotics $(6 \mu \mathrm{g} / \mathrm{ml} \mathrm{G} 418 ; 16 \mu \mathrm{g} / \mathrm{ml} \mathrm{hygromycin} \mathrm{B;10} \mu \mathrm{g} / \mathrm{ml}$ blasticidin; $10 \mu \mathrm{g} / \mathrm{ml}$ puromycin) on solid M199 media.

Immunofluorescence. A total of $1.5 \times 10^{6}$ promastigotes in the log phase of growth were fixed with $3 \%$ paraformaldehyde for $20 \mathrm{~min}$ at room temperature. The fixed cells were adhered to polyD-lysine (Millipore)-coated glass slides and permeabilized with $0.2 \%$ Triton X-100 for 5 min. The cells were blocked with 5\% skim powder milk in TBS-T for $1 / 2 \mathrm{~h}$. An anti-HA (Sigma H3663) primary antibody was diluted in $2.5 \%$ milk with TBS-T at 1:500 and incubated with the samples for 2 h. Secondary antibodies conjugated to Alexa Fluor 488 (Invitrogen A11001) at a 1:500 dilution in TBS were added and incubated for $1 / 2 \mathrm{~h}$. Hoechst (Invitrogen H3570) at a concentration of $2 \mu \mathrm{g} / \mathrm{ml}$ in TBS was used to stain the nuclei and kinetoplasts for 15 minutes. Images were acquired with an Axio Observer combined with an LSM 780 confocal device at 630x magnification (Carl Zeiss). Images were processed with Fiji ImageJ free software.

\section{Immunoprecipitation}

A total of $5 \times 10^{8}$ parasites in the $\log$ phase of growth were centrifuged $\left(2000 \mathrm{~g}, 10 \mathrm{~min}, 4^{\circ} \mathrm{C}\right)$, and the pellets were washed with $20 \mathrm{ml}$ of ice-cold PBS and centrifuged. The resulting pellets were resuspended in $1 \mathrm{ml}$ of lysis buffer (Tris-Cl pH $8.020 \mathrm{mM}, \mathrm{NaCl} 150 \mathrm{mM}$, Triton X-100 0.5\%, EDTA $0.5 \mathrm{mM}$, cOmplete mini [Roche] 3X), frozen in $\mathrm{N}_{2}$ and thawed on ice. A $21 \mathrm{G}$ needle was used to resuspend the suspension 20 times on ice to lyse the parasites, and $4 \mathrm{U}$ of Turbo DNAse was added to each sample. The samples were centrifuged $\left(10000 \mathrm{~g}, 10 \mathrm{~min}, 4^{\circ} \mathrm{C}\right)$, and the pellets were discarded. The supernatants were incubated with $30 \mu \mathrm{l}$ of protein A/G-coated magnetic beads (Genscript L00277) to remove the nonspecifically bound proteins for 1 hour at $4{ }^{\circ} \mathrm{C}$ with agitation. Ten micrograms of specific antibodies (anti-HA [Sigma H3663] or anti-MME [CST - 8015S]) were diluted in $500 \mu \mathrm{l}$ of lysis buffer and incubated with $100 \mu \mathrm{l}$ of the beads for 1 hour at room temperature with agitation. The unbound antibodies were removed using a magnet, and the beads 
were incubated with precleared supernatants for 2 hours at $4{ }^{\circ} \mathrm{C}$ with agitation. The beads were washed 6 times with $1 \mathrm{ml}$ of $0.05 \%$ TBS Tween using a magnet, and the bound proteins were eluted by boiling the beads with $60 \mu$ l of sample buffer $2 \mathrm{X}(117 \mathrm{mM}$ Tris-Cl pH $6.8,10 \%$ glycerol, $3.3 \%$ SDS, $0.04 \mathrm{mg} / \mathrm{mL}$ bromophenol blue, $2 \% 2$-mercaptoethanol) for 5 minutes. A fraction of the eluted proteins were used for western blotting, and the remaining proteins were sent to the University of Laval for mass spectrometry identification.

\section{Proteomic analysis}

The data obtained by mass spectrometry were analyzed using Scaffold 4 software (http://www.proteomesoftware.com/products/scaffold/). In the case of the anti-HA IP, the proteins identified in the WT sample (negative control) with a $\geq 50 \%$ probability of being correctly identified were removed from the analysis. After this removal, the criterion for identifying proteins that interacted with each PRMT was $\geq 95 \%$ protein identification probability. Interactivenn (38) was used to generate a Venn diagram. Tools of TriTrypDB (https://tritrypdb.org/tritrypdb/app) were used to search for proteins among the PRMT interacting proteins that harbor RGG motifs or gene ontology terms related to RNA binding. For the MME IP analysis, proteins whose normalized total spectra were at least 2 times higher in $\Delta 1$ were listed.

\section{THP-1 culture and differentiation}

THP-1 cells were cultured in complete RPMI-1640 medium (Sigma-Aldrich) supplemented with $10 \%$ heat-inactivated fetal bovine serum (Sigma-Aldrich) and $0.5 \%$ penicillin-streptomycin (Thermo Fisher Scientific) and incubated at $37^{\circ} \mathrm{C}$ in a $5 \% \mathrm{CO}_{2}$ atmosphere. The cells were not allowed to exceed a maximum density of $10^{6}$ cells $\cdot \mathrm{mL}^{-1}$. For cell differentiation, THP-1 monocytes were incubated with $30 \mathrm{ng} \cdot \mathrm{mL}^{-1} 4 \alpha$-phorbol 12-myristate 13-acetate - PMA (Sigma Aldrich) for 24 hours (39). After the differentiation period, the medium with nondifferentiated cells was removed, and the cells were incubated in fresh RPMI-1640 medium for 48 hours for complete maturation and improvement of phagocytic properties (40); then, the cells were infected with

\section{Leishmania.}

\section{In vitro infection assay}

The infection assays were performed at $33{ }^{\circ} \mathrm{C}$ in a $5 \% \mathrm{CO}_{2}$ atmosphere using differentiated THP1 macrophage-like host cells seeded in 96-well black flat-bottom plates at $2 \cdot 10^{4}$ cells $\cdot \mathrm{mL}^{-1}$. After 
the macrophage differentiation and maturation period, the THP-1 differentiated macrophage-like cells were infected with metacyclic promastigotes (10 parasites:1 host cell) purified during the stationary growth phase by Ficoll density gradient (41). After 4 hours of incubation, noninternalized parasites were removed by washing the cells with RPMI-1640 medium, and the plates were incubated at $33{ }^{\circ} \mathrm{C}$ in a $5 \% \mathrm{CO}_{2}$ atmosphere in fresh medium with $0.25 \%$ Hoechst (Merck-Millipore) to stain the DNA. At 24 and 48 hours postinfection, Leishmania intracellular growth was monitored by analyzing fluorescent images acquired by the ImageXpress Micro XLS System ${ }^{\circledR}$ (Molecular Devices, LLC, EUA); 9 fields of view of each well plate were examined under 40x magnification. DAPI filters ( $\lambda_{\mathrm{ex}}: 377$ and $\left.\lambda_{\mathrm{em}}: 447\right)$ and Texas Red filters $\left(\lambda_{\mathrm{ex}}: 562\right.$ and $\lambda_{\text {em: }}$ 624) were used to image the cell nuclei and Leishmania amastigotes, respectively. The cell and parasite counts were obtained using the algorithm Transfluor from MetaXpress Software (Version 5.3.0.5, Molecular Devices, LLC) by analysis of the acquired images. To count the nuclei, the algorithm was set to detect structures between 3-9 $\mu \mathrm{m}$ and intensities 1600 gray levels above background, while structures between 2.5-5 $\mu \mathrm{m}$ and intensities 1700 gray levels above background were used to count Leishmania (vesicles). The infection rate (\%) was calculated based on the ratio of infected cells to the total THP-1 cells counted, and statistical analysis (Dunnett method) was performed to identify significant differences in infection and/or growth between the control and knockout parasites.

For BMDM infection, bone marrow was harvested from BALB/c mouse femurs and tibias by cutting both ends of the bones and flushing out the marrow with PBS. Precursor cells were cultured in complete RPMI supplemented with $30 \%$ L929 cell-conditioned medium. After 7 days in culture, the mature BMDMs were harvested by washing the cells with cold PBS, incubating on ice for 15 min and pipetting up and down several times. One day prior to infection, $1 \times 10^{5}$ cells were plated on $13-\mathrm{mm}$ diameter coverslips and incubated at $37{ }^{\circ} \mathrm{C}$ in $5 \% \mathrm{CO}_{2}$ for $16 \mathrm{~h}$ in complete RPMI. The cells were infected with metacyclic promastigotes at an MOI of 1:1 for $4 \mathrm{~h}$ and washed with incomplete RPMI to remove free parasites. The cells were incubated in fresh complete RPMI for $24 \mathrm{~h}$ and $72 \mathrm{~h}$. Coverslips were collected at each time point, stained using the Diff-Quick method (LaborClin, Brazil) and mounted onto glass slides. The slides were analyzed under light microscopy, and 100 macrophages in each of the replicates were counted on each coverslip. 


\section{Evaluation of amastigote growth in culture}

Leishmania braziliensis M2903 pT_Tomato parental cells or PRMT-null mutants were seeded at $5 \times 10^{5}$ cells $/ \mathrm{mL}$ in $10 \mathrm{ml}$ of complete M199 medium and incubated at $26{ }^{\circ} \mathrm{C}$ for five days until they reached the stationary phase of growth. On the fifth day post inoculum, metacyclic promastigotes were isolated by Ficoll gradient (41) and counted using a Neubauer chamber. Metacyclic promastigotes were transferred to $10 \mathrm{ml}$ of fetal bovine serum (FBS) at $2 \times 10^{5}$ cells $/ \mathrm{ml}$ and incubated in vented flasks in a humid atmosphere at $33{ }^{\circ} \mathrm{C}$ in $5 \% \mathrm{CO}_{2}$ to undergo amastigogenesis. To verify the cell viability of the different PRMT-KO cell lines during the process of amastigogenesis, two milliliters of each culture was harvested every $24 \mathrm{~h}$ for 4 consecutive days, centrifuged at 1,300 xg for $10 \mathrm{~min}$, washed once with PBS and resuspended in $1 \mathrm{ml}$ of M199 containing $1 \mathrm{mg} / \mathrm{ml}$ MTT ((3-(4,5-dimethylthiazol 2-yl)-2,5-diphenyltetrazolium bromide). Next, $200 \mu \mathrm{l}$ of the cell suspensions were added to each well of 96-well plates (4 wells per cell line) and incubated at $26^{\circ} \mathrm{C}$ for $24 \mathrm{~h}$. The rate of MTT conversion (i.e., the increase in absorbance at $570 \mathrm{~nm}$ ) was used in a linear regression in GraphPad Prism 6, and the slope of the line was used as a measure of the relative amastigote growth in culture. At the end of the fourth day of culture in FBS, $500 \mu \mathrm{l}$ of the fully differentiated amastigotes were inoculated in $4.5 \mathrm{ml}$ of FBS (first passage) and incubated at $33{ }^{\circ} \mathrm{C}$ for four days. Then, the amastigotes were subjected to a second passage in FBS for four more days. At the end of the 12th day, $500 \mu 1$ of the same culture was inoculated into $4.5 \mathrm{ml}$ of M199 to evaluate for their ability to generate viable procyclic promastigotes. After $48 \mathrm{~h}$ of culture in M199, the number of procyclic cells was quantified by counting in a Neubauer chamber.

\section{Acknowledgements}

We thank Tania P. A. Defina for the technical support. We thank Eva Gluenz (University of Glasgow) for providing us with the plasmids for performing CRISPR/Cas9, and we thank Pegine Walrad and Michael Plevin (University of York) for the very productive discussions.

\section{Financial support}

This project was supported by Fundação de Amparo à Pesquisa do Estado de São Paulo, FAPESP (2018/14398-0, 2015/13618-8) grants to AKC; Brazilian National Council for Scientific and Technological Development (https://www.gov.br/cnpq/pt-br), CNPq (305775/2013-8) to AKC. This study was supported in part by the Coordenação de Aperfeiçoamento de Pessoal de Nível Superior - Brasil (CAPES - https://www.gov.br/capes/pt-br) - Finance Code 001, AKC. T.R.F. is 
funded by the Intramural Research Program of the National Institute of Allergy and Infectious Diseases, National Institutes of Health. During the course of this work, LBL (grant 2016/009690), JAD (grant 2016/14657-0), JCQJ (grant 2020/00088-9), GDC (grant 2020/02372-6), LA (grant 2017/19040-3) and RDMM (grant 2019/18607-5) were supported by FAPESP (Fundação de Amparo a Pesquisa do Estado de São Paulo) fellowships. FPV was supported by CAPES.

\section{Ethics statement}

All mouse experimental procedures were performed in accordance with the Ethical Principles in Animal Research approved by the Local Ethical Animal Committee (CEUA) of FMRP-USP (protocol 107/2020).

The funders had no role in study design, data collection and analysis, decision to publish, or preparation of the manuscript. 
bioRxiv preprint doi: https://doi.org/10.1101/2021.09.22.461376; this version posted September 22, 2021. The copyright holder for this preprint (which was not certified by peer review) is the author/funder, who has granted bioRxiv a license to display the preprint in perpetuity. It is made available under aCC-BY-NC-ND 4.0 International license.

\section{Figures}

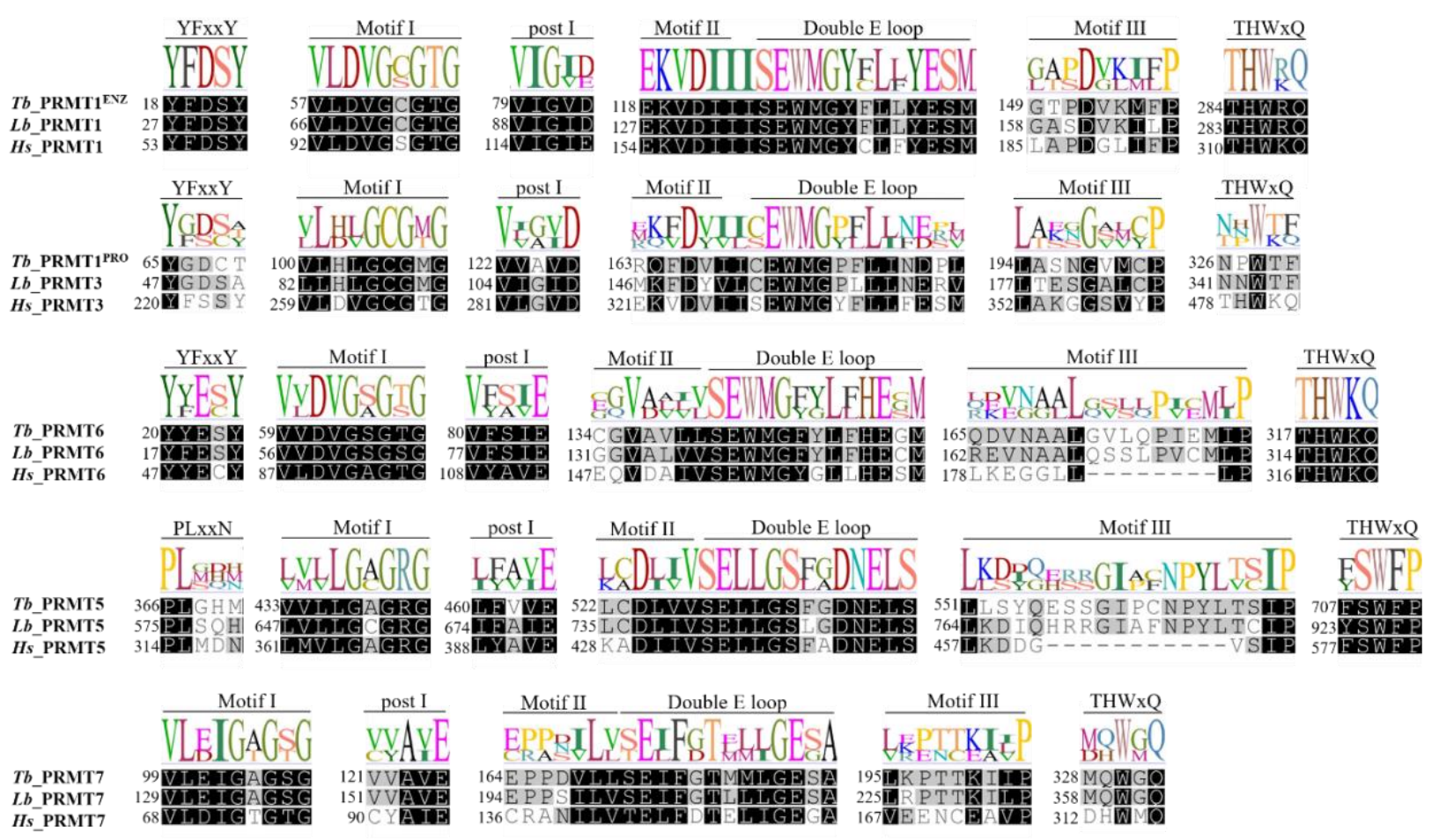

Figure 1. Global sequence alignment of PRMTs from L. braziliensis, T. brucei and Homo sapiens. The protein sequences were aligned using Geneious v6.18 software with Clustal Omega. The key motifs of the conserved PRMT methyltransferase domain are indicated. Residue shading: gray background: 60-80\% similar; black background: $80-$ $100 \%$ similar. The amino acid sequence logo is shown on the top of each alignment representing motif sequence conservation. 


\section{a}
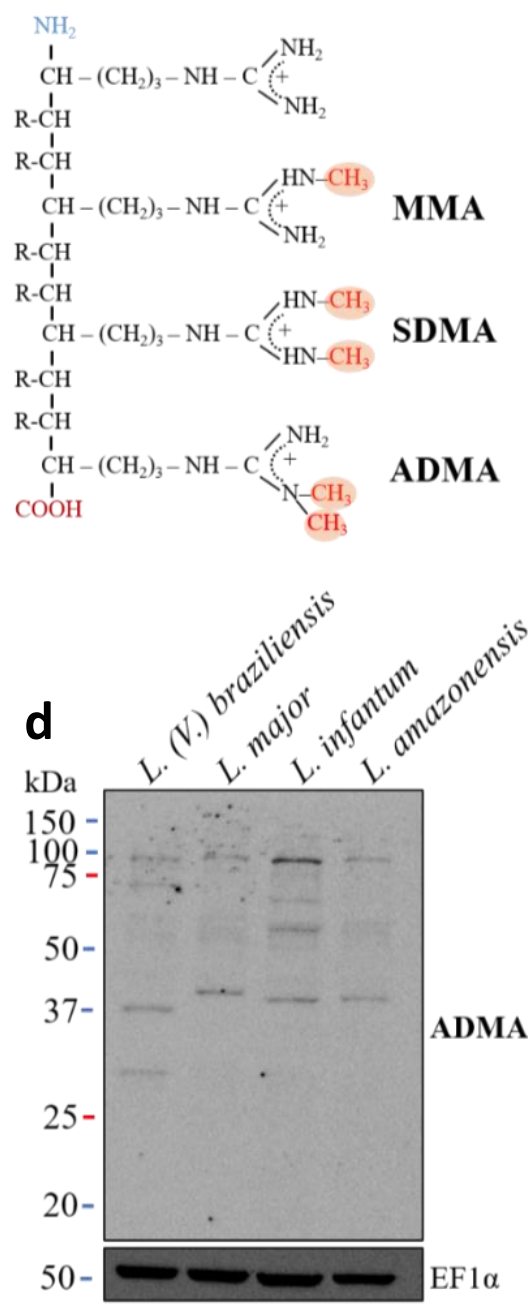

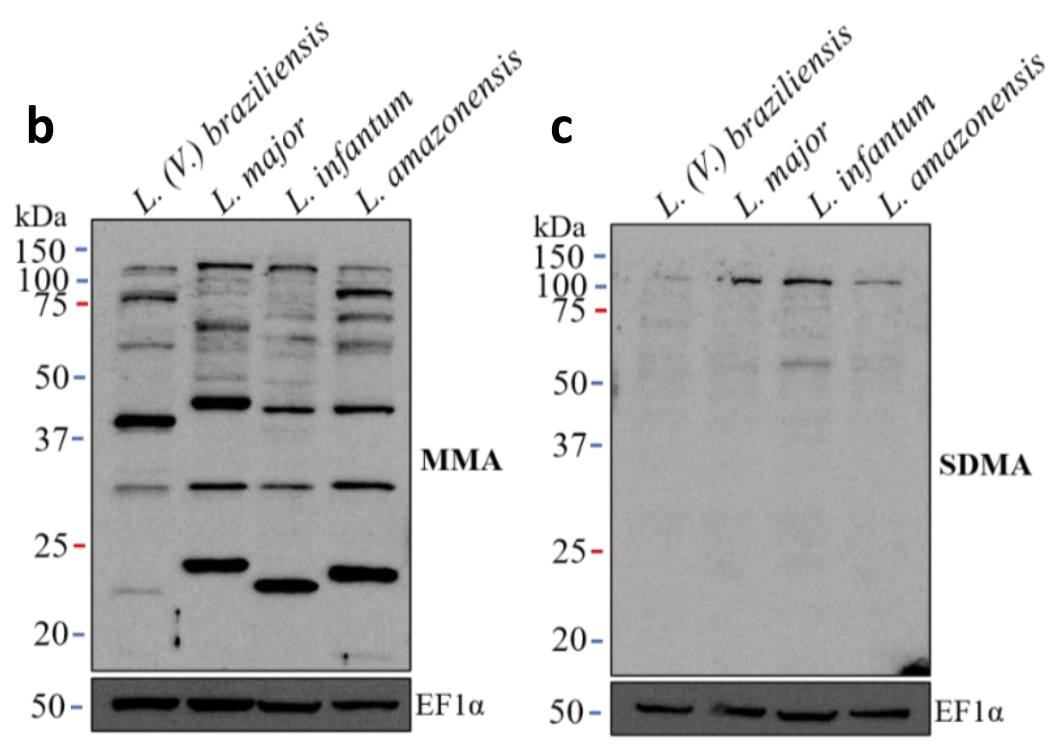

e

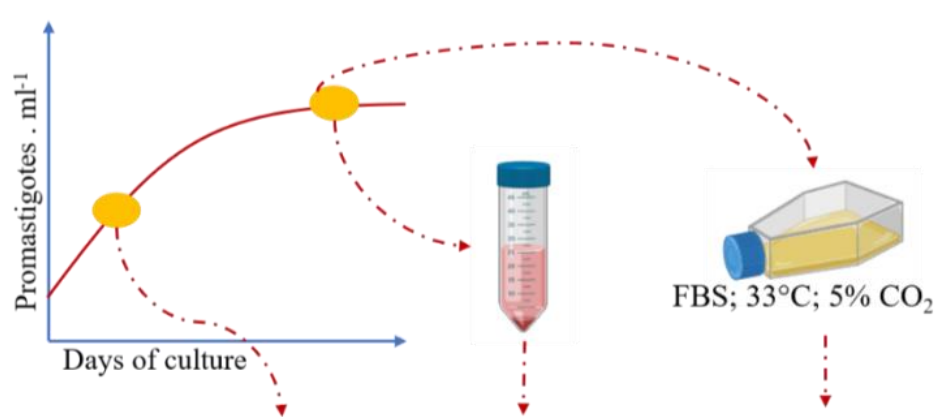

Procyclic Promastigotes

Metacyclic Promastigotes

Axenic Amastigotes
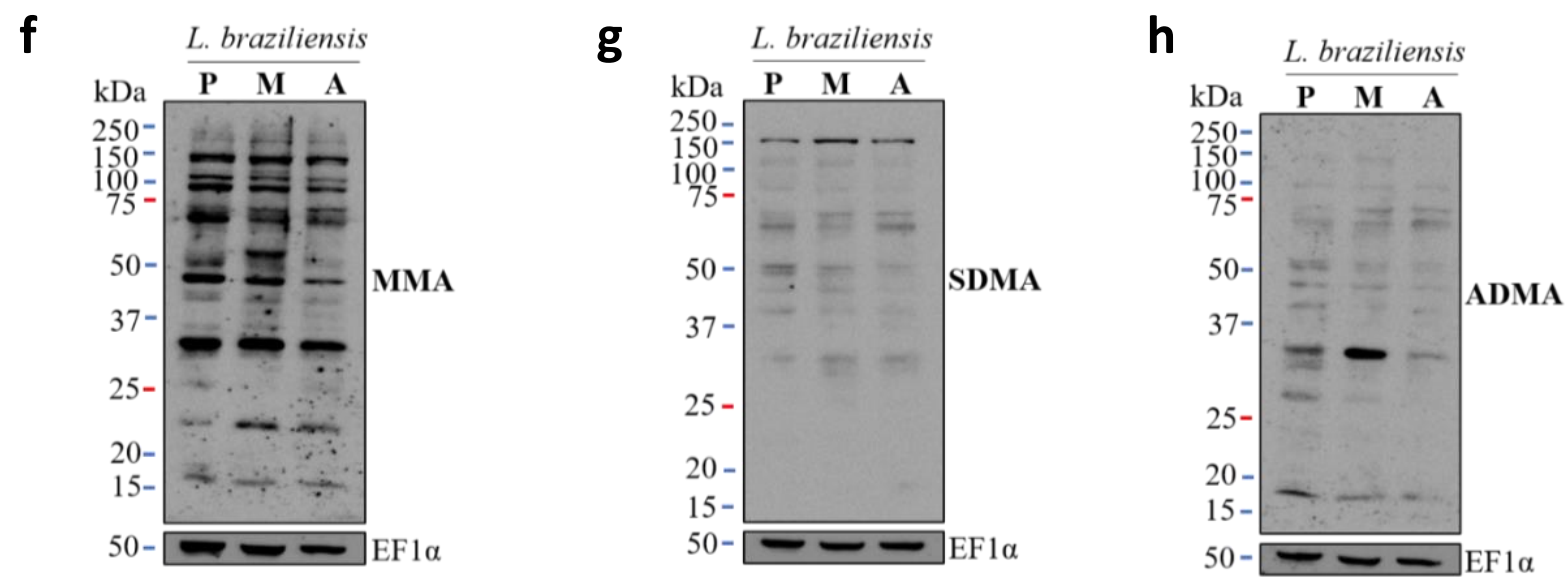

Figure 2. Arginine methylation is present in different Leishmania species and stages of the Leishmania life cycle. a. Schematic representation of the three major types of protein arginine methylation catalyzed by PRMTs. b, $\mathbf{c}$ and $\mathbf{d}$. 
The profiles of monomethylated (MMA), symmetrically dimethylated (SDMA) and asymmetrically dimethylated (ADMA) arginine in different species of Leishmania were determined by western blotting. e. A schematic representation of the protocol used to obtain samples from three distinct stages of the L. braziliensis life cycle. $\mathbf{f}, \mathbf{g}$ and $\mathbf{h}$. The profiles of MMA, SDMA and ADMA of distinct stages of the L. braziliensis life cycle; (P) promastigotes, (M) metacyclic promastigotes and (A) amastigotes. The loading control for all the western blots (Panels b, c, d, f, $\mathbf{g}$ and $\mathbf{h}$ ) is shown on the lower blots using the anti-EF1alpha antibody.

a

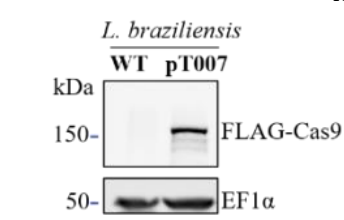

C

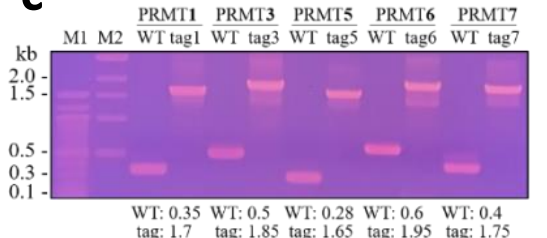

d

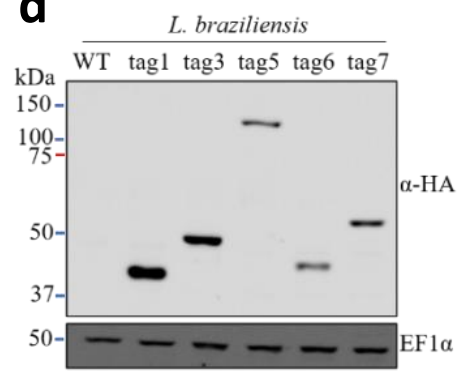

e b
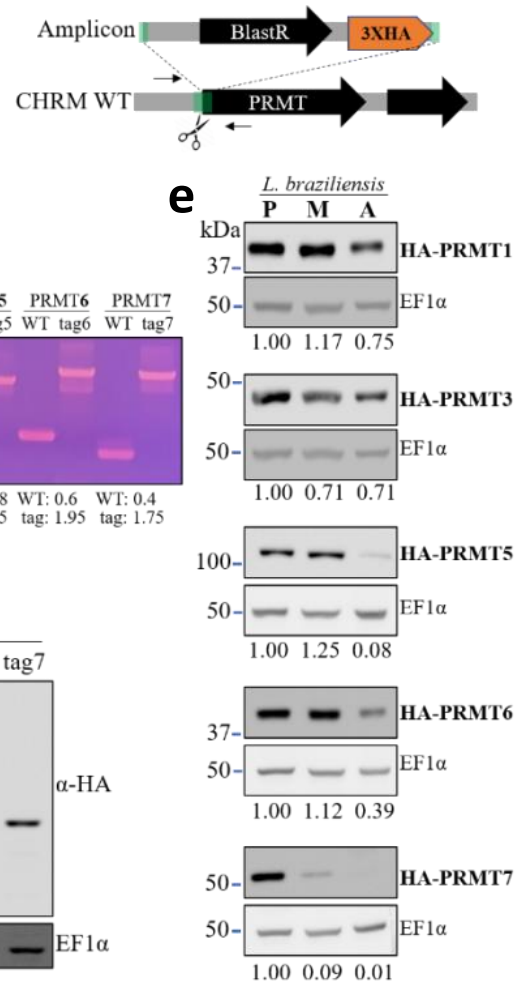

f

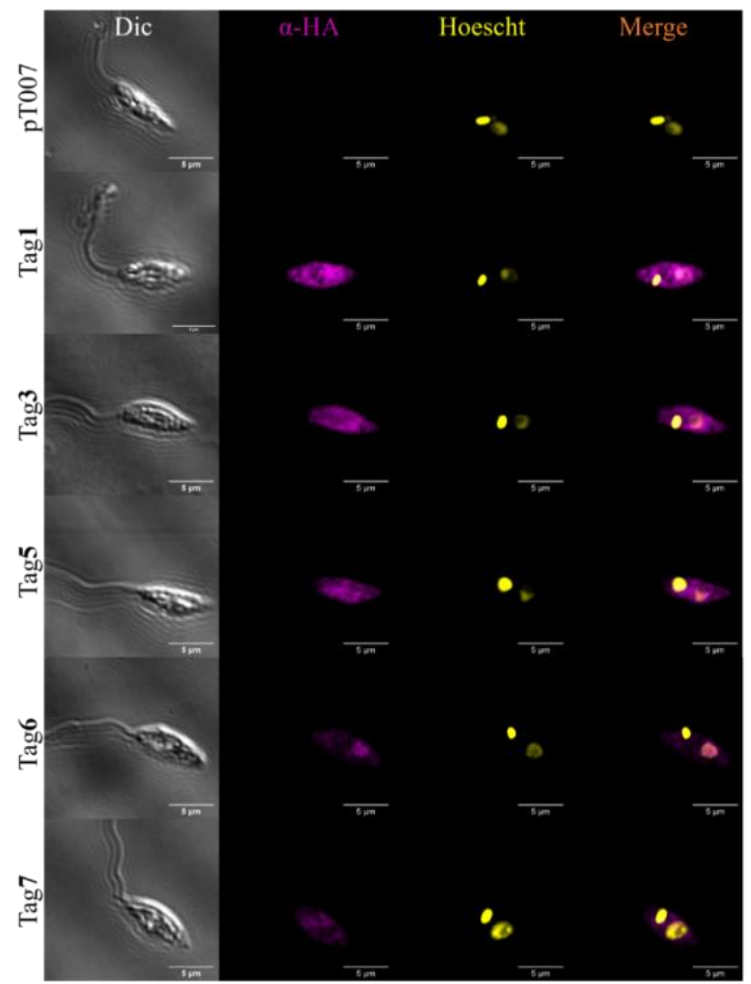

Figure 3. HA-tagged PRMTs are preferentially expressed in promastigotes, and most of them are cytoplasmic. a. Expression of FLAG-Cas9 in L. braziliensis pT007, as shown by western blotting with anti-FLAG antibodies (Sigma F1804). b. Schematic representation of the strategy used for HA tagging of the N-terminus of each PRMT coding gene. BlastR represents the blasticidin resistance gene; the black arrow refers to the PRMT coding sequence in the respective genomic locus (CHRM: chromosome), and the green boxes represent a 30-bp homology to the locus to be edited; the thin black arrows depict the annealing site of the primers used in the diagnostic PCR shown in $c$. c. Confirmation of PRMT endogenous HA tagging by PCR analysis and agarose gel (1\%) electrophoresis showing the expected amplicon length for each of the tagged PRMTs for wild-type (WT) and tagged PRMTs (tag) in kilobase pairs. M1 and M2 are standard MW markers (100 bp and $1 \mathrm{~kb}$, respectively - both from New England Biolabs). d. Expression of HA-tagged PRMTs (tag1 indicates HA-PRMT1; tag3 indicates HA-PRMT3, and so on) in $L$. braziliensis procyclic promastigotes. The blots confirm the expected molecular weight for each PRMT. In the upper panel, an anti-HA antibody (H3663 - Sigma Aldrich) was used. e. Evaluation of HA-PRMT expression throughout development; procyclic promastigotes $(\mathrm{P})$, metacyclic promastigotes $(\mathrm{M})$ and axenic amastigotes (A) of $L$. braziliensis. The values below the panels represent the densitometric analysis (PRMT/EF1 $\alpha$ ). f. Confocal microscopy showing the subcellular localization of HA-PRMTs. An anti-HA antibody (H3663 Sigma) was used to detect the HAPRMTs, while Hoechst 33342 (Invitrogen) was used to detect the mitochondrial and nuclear DNA. EF1alpha was evaluated as a loading control in all the western blotting data shown. 

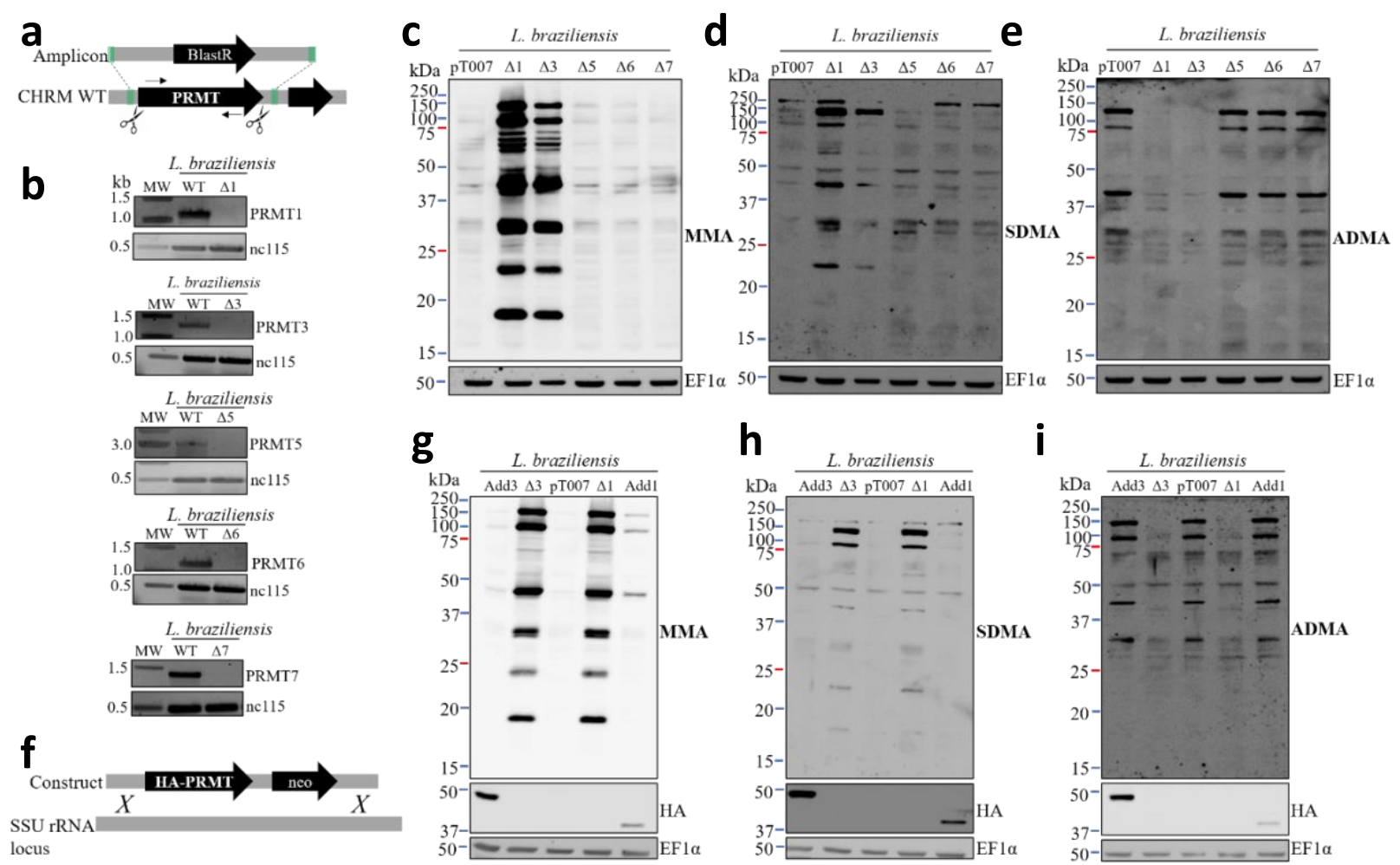

Figure 4. Gene knockout of PRMT1 and PRMT3 leads to changes in arginine methylation profiles. a. Schematic representation of the strategy used to generate PRMT-null mutants (black arrows represent the primers used in PCR shown in $b$ ). b. Confirmation of gene deletion in the transfectant clones by PCR using primers that anneal within the PRMT CDS. c, d and e. Arginine methylation profiles of monomethylated (MMA), symmetrically dimethylated (SDMA) and asymmetrically dimethylated arginine (ADMA) in null mutants ( $\Delta n$ represents PRMT $n$ gene deletion) were determined by Western blotting. f. Schematic representation of the strategy used to generate PRMT addback parasites. $\mathbf{g}, \mathbf{h}$ and $\mathbf{i}$. Arginine methylation profiles of MMA, SDMA and ADMA of addback parasites (Add $n$ represents PRMT $n$ addback in a $\Delta n$ background) were determined by Western blotting. CHRM: chromosome; MW: DNA molecular weight marker; nc115 and EF1a were used as loading controls in DNA agarose gels and protein blots, respectively. 

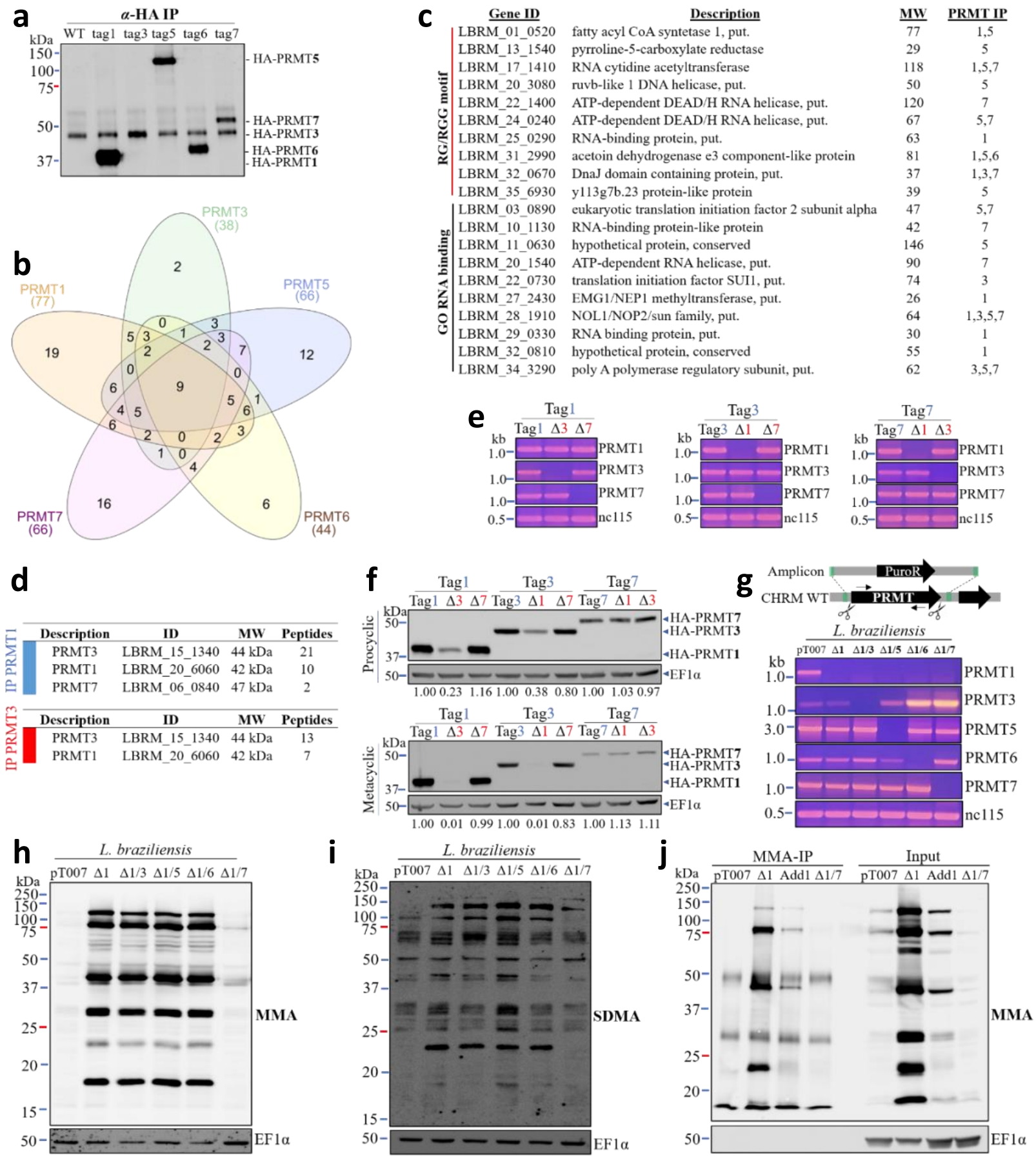

Figure 5. The PRMT interactome has diverse RNA-binding proteins and suggests interplay between PRMTs. a. Validation of HA-PRMT immunoprecipitation (IP) by Western blotting with anti-HA antibodies (Tag $n$ represents HA-tagged PRMTn). b. Venn diagram of the proteins co-IP with each of the HA-PRMTs. c. List of the PRMT interacting proteins that either have RGG motifs or are associated with GO terms related to RNA binding (MW: molecular weight in $\mathrm{kDa}$, T: true, F: false for the presence of an RG motif or association with an RBP-related GO term) d. List of PRMTs interacting with each other as detected in the co-IP assays. e. Confirmation of PRMT1/3/7 gene deletion in HA-tagged transfectants by PCR. f. Effects of PRMT gene deletion on the expression levels of HAPRMT1/3/7 as evaluated by Western blotting. g. Confirmation of double PRMT gene knockout by PCR; diagram represents the strategy used to generate the double null mutants. $\mathbf{h}$ and $\mathbf{i}$. Arginine methylation profiles of 
monomethylated (MMA) and symmetrically demethylated (SDMA) in double-null parasites as determined by Western blotting. j. Monomethyl arginine (MMA) IP. Western blotting analysis of MMA profiles of proteins immunoprecipitated using an anti-MMA antibody. CHRM WT: wild-type chromosome; nc115 and EF1a were used as loading controls in DNA agarose gels and protein blots, respectively.
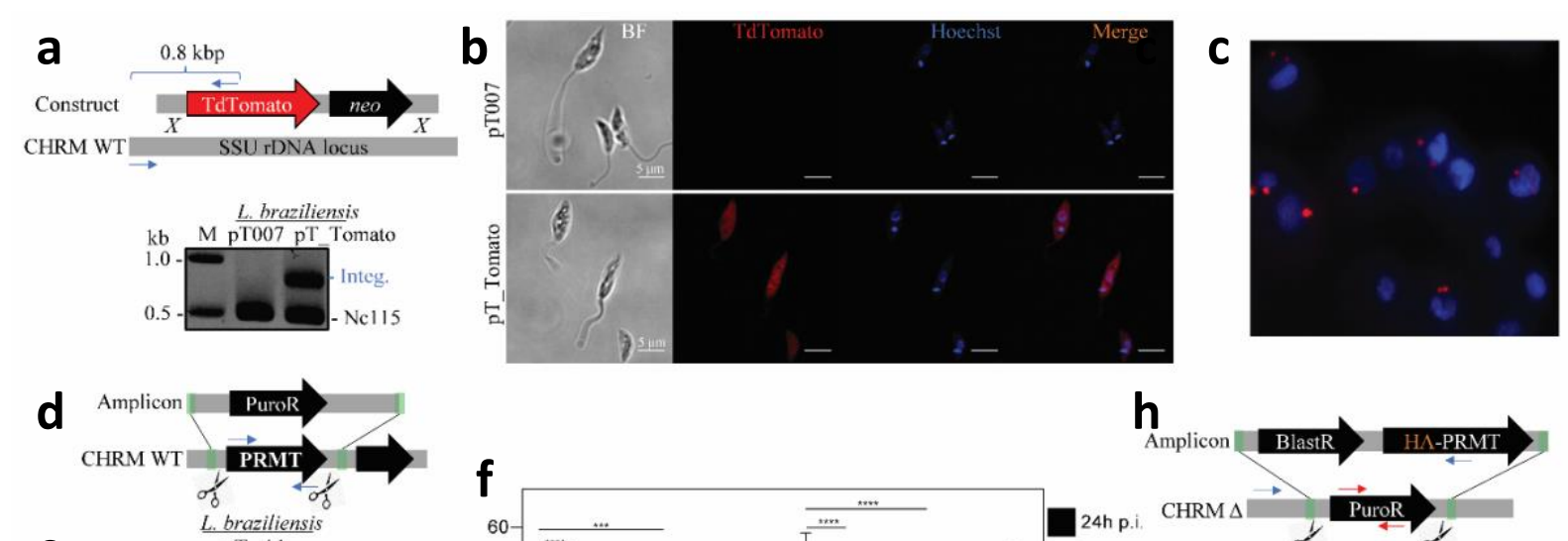

e
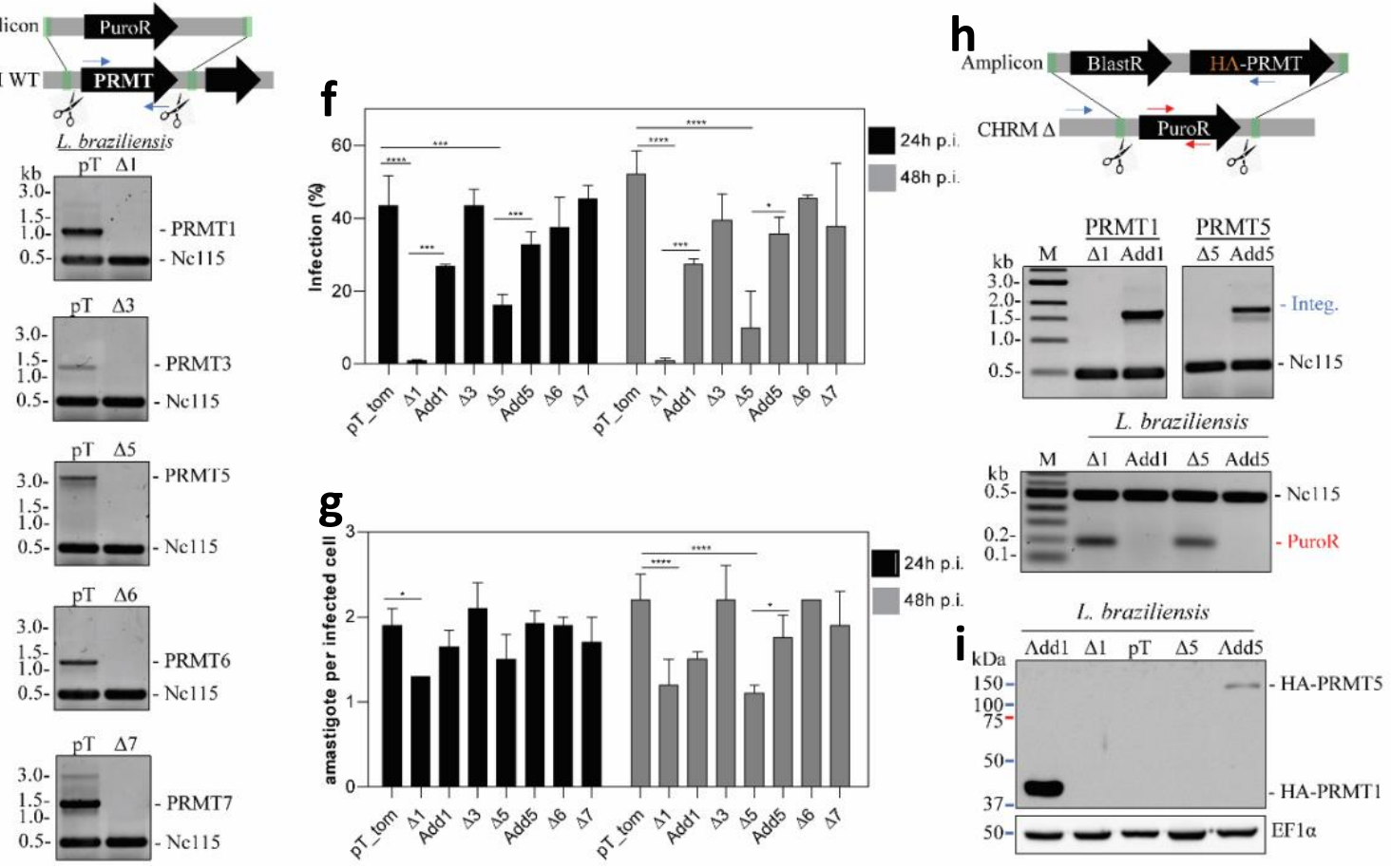

Figure 6. PRMT1- and PRMT5-knockout parasites fail to efficiently infect human macrophages in vitro. a. Generation of L. braziliensis expressing Cas9 (pT) and Tdtomato (tom). Upper panel: schematic representation of the strategy used to integrate the Td_tomato gene into the SSU rDNA locus of L. braziliensis pT007. Blue arrows represent primers used to confirm the integration (integ.) of the Td_tomato gene into the SSU rDNA locus; Lower panel: gel electrophoresis of the product of the diagnostic multiplex PCR to confirm Tdtomato integration into the ribosomal rRNA genomic locus (Integ.). b. Confocal fluorescence microscopy of the pT007 and pT_tomato parasites. c. Representative image of an in vitro infection of THP-1 cells with the pT_tomato strain. The images were acquired by ImageXpress Micro XLS System ${ }^{\circledR}$, as described in the methods section. d. Strategy to knockout PRMTs using pT_tomato parasites; blue arrows represent primers used to detect the presence of PRMT CDSs. e. Gel electrophoresis of the products of the diagnostic multiplex PCR of the null mutants. $\mathbf{f}$ and $\mathbf{g}$. In vitro infection of THP-1 macrophages evaluated by assessing the percentage of infected cells and the mean number of amastigotes per infected cell. $\mathbf{h}$. Generation of addback transfectants. Upper panel: schematic representation of the strategy used to integrate HAtagged PRMT1 and PRMT5 into the former loci. Lower panels: Gel electrophoresis of the products of the diagnostic multiplex PCR of addback transfectants. Blue arrows indicate primers used to confirm the integration of HA-PRMT1 and 5; red arrows indicate primers used to detect the presence of PuroR. i. Expression of PRMT1 and PRMT5 in the 
bioRxiv preprint doi: https://doi.org/10.1101/2021.09.22.461376; this version posted September 22, 2021. The copyright holder for this preprint (which was not certified by peer review) is the author/funder, who has granted bioRxiv a license to display the preprint in perpetuity. It is made available under aCC-BY-NC-ND 4.0 International license.

addback transfectants (western blotting). Nc115: unrelated gene used as a loading control of the multiplex PCRs (a, d and h). CHRM WT: wild-type chromosome. Experiments were performed in biological triplicates. $* * p<0.002$, *** $p<0.0002$ and $* * * * p<0.0001$, ANOVA Dunnett's test. 


\section{a}
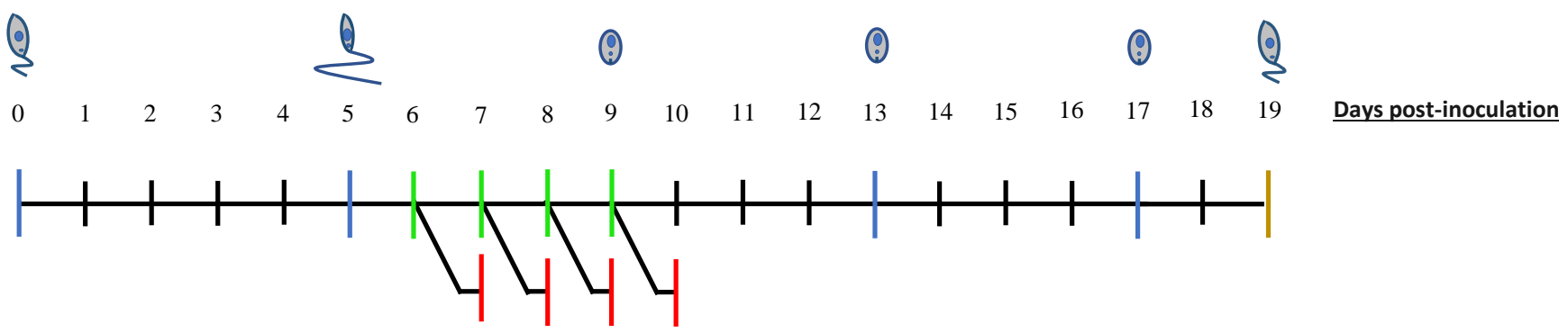

b

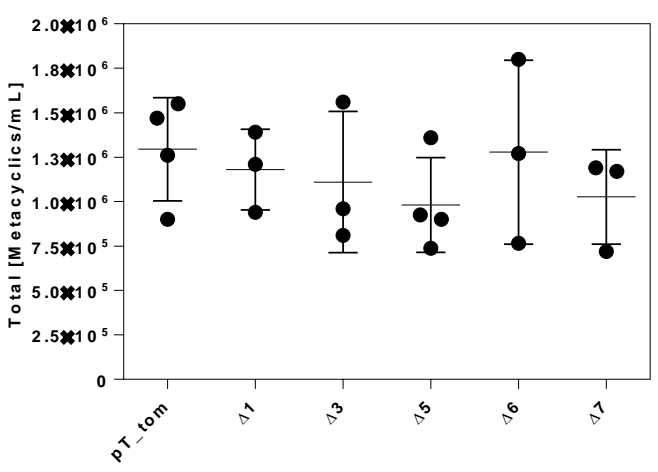

C

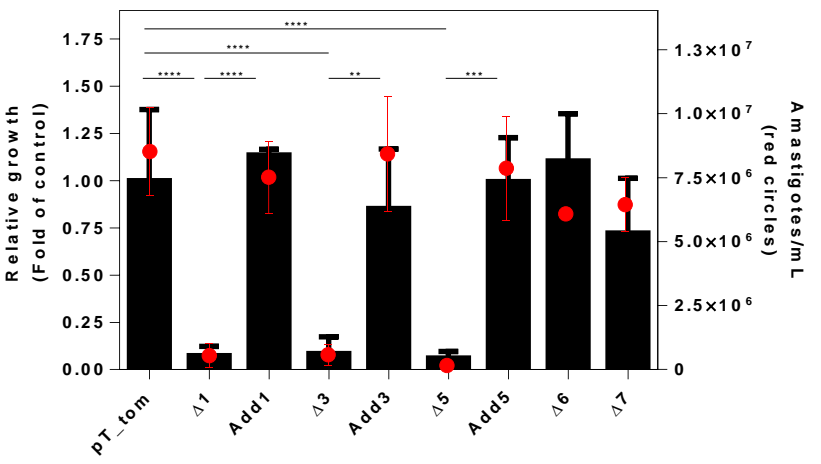

Figure 7. Knockout of LbPRMT1, LbPRMT3 and LbPRMT5 impairs Leishmania braziliensis axenic amastigote proliferation in vitro. A) Schematic representation of the experimental design to assess the viability and proliferation of PRMT-null mutants of axenic amastigotes in vitro compared with pT_Tomato parental cells (WT). On the fifth day post-inoculum, metacyclic promastigotes were isolated by Ficoll gradient, counted and transferred to fetal bovine serum to undergo amastigogenesis. Parasites were collected every $24 \mathrm{~h}$ for 4 consecutive days (green lines) and incubated in medium containing MTT for $24 \mathrm{~h}$ to determine cell viability (red lines). Amastigotes were subjected to two passages in FBS. At the end of the $12^{\text {th }}$ day of culture, the amastigotes were transferred into complete medium to evaluate their ability to generate viable procyclic forms (yellow line). B) Concentration of metacyclic promastigotes at day 5 post-inoculum. C) The absorbance values after MTT conversion were used in a linear regression (utilizing GraphPad Prism 6) to determine the relative growth of each cell line ( $n \geq 3$ ). The slope of each line was used as a measure of growth and viability. At the end of the fourth day of differentiation, the number of amastigotes was determined (red circles) using a Neubauer chamber $(n \geq 2)$. ** $\mathrm{P}<0.01, * * * \mathrm{P}<0.005$, $* * * * \mathrm{P}<0.001$ by one-way ANOVA followed by Tukey's test. 
bioRxiv preprint doi: https://doi.org/10.1101/2021.09.22.461376; this version posted September $22,2021$. The copyright holder for this preprint (which was not certified by peer review) is the author/funder, who has granted bioRxiv a license to display the preprint in perpetuity. It is made available under aCC-BY-NC-ND 4.0 International license.

Table 1. List of the proteins that were at least two times more abundant in $\Delta 1$ IP.

$\quad$ Description
Put. centromere/microtubule binding protein cbf5
Uncharacterized protein
Contig, possible fusion of chromosomes 20 and 34
Put. small GTP-binding protein Rab1
Put. nuclear protein family a (Nop10p)
TDR1 protein
Uncharacterized protein
Acetyl-coenzyme A synthetase
SRP40_C domain-containing protein
Uncharacterized protein
Uncharacterized protein
Uncharacterized protein
Put. RNA helicase
PGFS protein
CP-type G domain-containing protein
Contig, possible fusion of chromosomes 20 and 34
RNA binding protein-like protein
Contig, possible fusion of chromosomes 20 and 34
Bac_surface_Ag domain-containing protein
Glutathione peroxidase
Put. coronin
Uncharacterized protein
NPL domain-containing protein
Put. small GTP-binding protein Rab18
Protein phosphatase methylesterase 1
Put. bystin
Uncharacterized protein
Uncharacterized protein
Put. prolyl oligopeptidase
Put. p22 protein
Small nuclear ribonucleoprotein Sm D2
Put. branched-chain amino acid aminotransferase

gene ID
LBRM_21_2070
LBRM_09_1060
LBRM_20_3610
LBRM_27_0850
LBRM_35_0430
LBRM_31_0550
LBRM_30_3080
LBRM_23_0800
LBRM_10_0100
LBRM_34_4360
LBRM_28_0950
LBRM_07_0220
LBRM_21_0670
LBRM_31_2410
LBRM_05_0200
LBRM_20_4990
LBRM_07_1050
LBRM_20_4440
LBRM_31_0220
LBRM_26_0830
LBRM_23_1260
LBRM_35_5930
LBRM_07_0670
LBRM_33_2110
LBRM_32_1000
LBRM_30_0560
LBRM_34_4820
LBRM_07_0680
LBRM_35_7100
LBRM_30_1090
LBRM_33_3460
LBRM_27_2210

MW
49
67
17
22
8
50
36
77
28
56
20
66
75
32
89
81
49
29
53
19
57
37
20
24
31
53
39
26
78
22
12
44

Normalized total spectra (Unique pep.)*

\begin{tabular}{|c|c|c|c|c|}
\hline pT & $\Delta 1$ & Add1 & $\Delta 1 / \Delta 7$ & RBP/RG \\
\hline $5.08(6)$ & $87.72(30)$ & $32.02(23)$ & $17.2(14)$ & RBP \\
\hline $5.92(5)$ & $14.84(9)$ & $6.79(6)$ & $3.82(2)$ & - \\
\hline $1.69(2)$ & $13.5(7)$ & $4.85(5)$ & $2.87(3)$ & - \\
\hline $1.69(2)$ & $9.45(5)$ & $2.91(2)$ & $3.82(4)$ & - \\
\hline $0.1(0)$ & $21.59(5)$ & $6.79(3)$ & $2.87(2)$ & RBP \\
\hline 3.39 (4) & $8.1(5)$ & $2.91(3)$ & $1.91(2)$ & - \\
\hline $2.54(2)$ & $6.75(5)$ & $2.91(3)$ & $1.91(2)$ & RBP \\
\hline $2.54(3)$ & $8.1(6)$ & $1.94(2)$ & $3.82(4)$ & - \\
\hline $0.1(0)$ & $10.8(5)$ & $3.88(2)$ & $0.96(1)$ & - \\
\hline $1.69(2)$ & $10.8(4)$ & 3.88 (4) & $0.96(1)$ & RBP/RG \\
\hline $0.1(0)$ & $9.45(5)$ & $1.94(2)$ & $0.96(1)$ & - \\
\hline $1.69(2)$ & $8.1(6)$ & $1.94(2)$ & $1.91(2)$ & - \\
\hline $1.69(2)$ & $4.05(3)$ & $1.94(2)$ & $1.91(2)$ & - \\
\hline $0.85(1)$ & $2.7(2)$ & $0.97(1)$ & $0.96(1)$ & - \\
\hline $0.1(0)$ & $2.7(2)$ & $0.97(1)$ & $0.96(1)$ & - \\
\hline $1.69(2)$ & $4.05(3)$ & $0.97(1)$ & $0.1(0)$ & - \\
\hline $0.85(1)$ & $6.75(4)$ & $2.91(3)$ & $0.1(0)$ & RBP \\
\hline 0.85 (1) & $4.05(2)$ & 0.97 (1) & $0.96(1)$ & - \\
\hline $0.85(1)$ & $2.7(2)$ & $0.97(1)$ & $0.96(1)$ & - \\
\hline $1.69(2)$ & $4.05(3)$ & $1.94(2)$ & $0.96(1)$ & - \\
\hline $0.1(0)$ & $4.05(2)$ & $1.94(2)$ & $0.96(1)$ & - \\
\hline $0.85(1)$ & $2.7(2)$ & $0.97(1)$ & $0.96(1)$ & - \\
\hline $0.1(0)$ & $8.1(5)$ & $0.97(1)$ & $0.1(0)$ & - \\
\hline $0.85(1)$ & $4.05(2)$ & $1.94(2)$ & $0.96(1)$ & - \\
\hline $0.85(1)$ & $2.7(2)$ & $0.97(1)$ & $0.96(1)$ & - \\
\hline $0.85(1)$ & $2.7(2)$ & $0.97(1)$ & $0.96(1)$ & - \\
\hline $0.85(1)$ & $2.7(2)$ & $0.1(0)$ & $0.96(1)$ & - \\
\hline $0.85(1)$ & $4.05(3)$ & $0.1(0)$ & $0.1(0)$ & - \\
\hline $0.1(0)$ & $2.7(2)$ & $0.1(0)$ & $0.96(1)$ & - \\
\hline $0.1(0)$ & $2.7(2)$ & $0.1(0)$ & $0.96(1)$ & - \\
\hline $0.85(1)$ & $2.7(2)$ & $0.1(0)$ & $0.96(1)$ & - \\
\hline $0.1(0)$ & $2.7(2)$ & $0.1(0)$ & $0.1(0)$ & - \\
\hline
\end{tabular}

Put.: Putative; MW: Molecular weight in kDa. *Normalized total spectra of each protein detected for each group; values inside parentheses are the number of unique peptides identified for each protein. RG/RBP represents proteins that either have RG motifs or are related to RNA-binding protein-related gene ontology terms.

Table 2. Proteins with R-methyl peptides identified in MMA IP

Description
Elongation factor 1-alpha
Tubulin beta chain
Put. heat-shock protein hsp70
Tubulin alpha chain
Put. ATP-dependent RNA helicase
Put. heat shock 70-related protein 1,mitochondrial
Chaperonin HSP60, mitochondrial
Put. ATP-dependent RNA helicase
Put. glycosomal phosphoenolpyruvate carboxykinase
Uncharacterized protein
Polyadenylate-binding protein
WD_REPEATS_REGION domain-containing protein
Activated protein kinase c receptor (LACK)
Put. ATP-dependent DEAD/H RNA helicase
40S ribosomal protein SA

$\begin{array}{cc}\text { gene ID } & \text { MW } \\ \text { LBRM_17_0090 } & 49 \\ \text { LBRM_33_0920 } & 50 \\ \text { LBRM_28_2990 } & 71 \\ \text { LBRM_13_0190 } & 50 \\ \text { LBRM_32_0470 } & 66 \\ \text { LBRM_30_2440 } & 71 \\ \text { LBRM_35_2240 } & 60 \\ \text { LBRM_34_3010 } & 94 \\ \text { LBRM_27_1920 } & 58 \\ \text { LBRM_25_2210 } & 32 \\ \text { LBRM_34_4130 } & 64 \\ \text { LBRM_06_0030 } & 73 \\ \text { LBRM_28_2950 } & 34 \\ \text { LBRM_07_0360 } & 65 \\ \text { LBRM_35_5370 } & 28\end{array}$




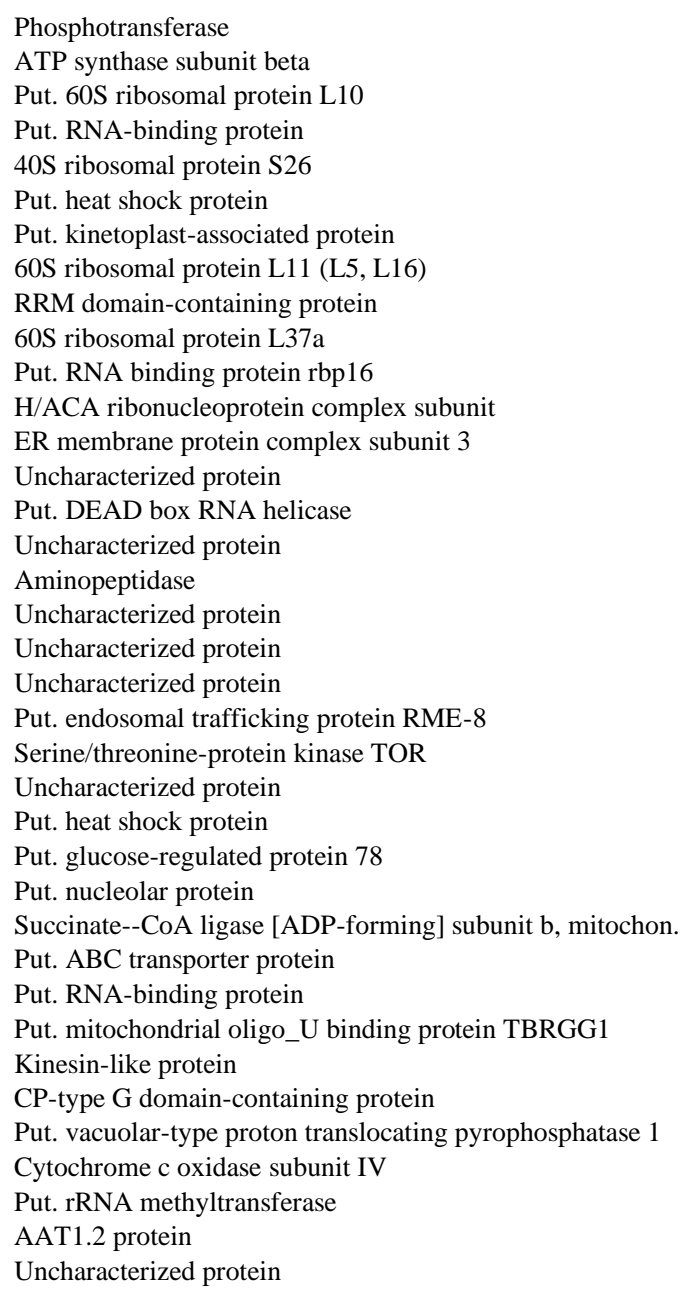

\begin{tabular}{|c|c|c|c|c|}
\hline LBRM_21_0310 & 52 & 259 & & \\
\hline LBRM_25_1150 & 56 & 384 & & \\
\hline LBRM_04_0950 & 25 & 55,128 & & \\
\hline LBRM_34_2130 & 30 & 163 & & RBP \\
\hline LBRM_30_3230 & 13 & 30 & & \\
\hline LBRM_33_2670 & 72 & 303 & & \\
\hline LBRM_35_6130 & 14 & 98 & & \\
\hline LBRM_04_0520 & 22 & 74 & & \\
\hline LBRM_25_0970 & 48 & 384,391 & & RBP/RG \\
\hline LBRM_33_0880 & 11 & 21 & & \\
\hline LBRM_28_0880 & 15 & 104 & & $\mathrm{RBP} / \mathrm{RG}$ \\
\hline LBRM_20_3910 & 22 & $190,198,206,214$ & & $\mathrm{RBP} / \mathrm{RG}$ \\
\hline LBRM_35_0450 & 29 & 147 & & \\
\hline LBRM_34_4360 & 56 & 392,396 & & $\mathrm{RBP} / \mathrm{RG}$ \\
\hline LBRM_35_2370 & 63 & 272 & & \\
\hline LBRM_25_2250 & 33 & 57 & & RBP/RG \\
\hline LBRM_29_2220 & 97 & 470 & & \\
\hline LBRM_32_2310 & 106 & $445,508,617,621,646$ & 436,644 & \\
\hline LBRM_34_3290 & 62 & 481 & & RBP \\
\hline LBRM_15_0570 & 75 & 372,375 & & \\
\hline LBRM_30_2160 & 272 & 1912 & & \\
\hline LBRM_35_6620 & 292 & 860,965 & 969 & RG \\
\hline LBRM_26_1530 & 154 & 47,740 & & \\
\hline LBRM_18_1400 & 92 & & 640 & \\
\hline LBRM_28_1300 & 72 & & 203 & \\
\hline LBRM_31_0300 & 70 & & 447 & $\mathrm{RBP}$ \\
\hline LBRM_35_3170 & 44 & & 406 & \\
\hline LBRM_03_0170 & 82 & & 315 & \\
\hline LBRM_29_0690 & 78 & & 505 & RBP/RG \\
\hline LBRM_30_0900 & 78 & & 515 & $\mathrm{RBP} / \mathrm{RG}$ \\
\hline LBRM_23_0710 & 83 & & 483 & \\
\hline LBRM_05_0200 & 89 & & 176 & \\
\hline LBRM_31_1450 & 84 & & 99 & \\
\hline LBRM_12_0670 & 39 & & 143 & \\
\hline LBRM_27_2160 & 105 & & 857 & \\
\hline LBRM_31_0440 & 55 & & 23 & \\
\hline LBRM_01_0710 & 80 & & 569 & \\
\hline
\end{tabular}

Put.: Putative; MW: Molecular weight in kDa. MMA and DMA residues: positions of the mono- and dimethylated arginine in each protein, respectively. Positions in yellow represent peptides with a probability of being correctly identified lower than $95 \%$ but higher than $88 \%$, and residues in black represent $\geq 95 \%$. RG/RBP represents proteins that either have RG motifs or are related to RNA-binding protein-related gene ontology terms.

\section{References}

1. Bedford MT, Clarke SG. Protein arginine methylation in mammals: who, what, and why. Mol Cell. 2009;33(1):1-13.

2. Yu MC, Lamming DW, Eskin JA, Sinclair DA, Silver PA. The role of protein arginine methylation in the formation of silent chromatin. Genes Dev. 2006;20(23):3249-54.

3. Al-Hamashi AA, Diaz K, Huang R. Non-Histone Arginine Methylation by Protein Arginine Methyltransferases. Curr Protein Pept Sci. 2020;21(7):699-712.

4. Jarrold J, Davies CC. PRMTs and Arginine Methylation: Cancer's Best-Kept Secret? Trends Mol Med. 2019;25(11):993-1009.

5. Jahan S, Davie JR. Protein arginine methyltransferases (PRMTs): role in chromatin organization. Adv Biol Regul. 2015;57:173-84.

6. Tewary SK, Zheng YG, Ho MC. Protein arginine methyltransferases: insights into the enzyme structure and mechanism at the atomic level. Cell Mol Life Sci. 2019;76(15):2917-32. 
7. Hughes RM, Waters ML. Arginine methylation in a beta-hairpin peptide: implications for Arg-pi interactions, DeltaCp(o), and the cold denatured state. J Am Chem Soc. 2006;128(39):12735-42.

8. Horowitz S, Trievel RC. Carbon-oxygen hydrogen bonding in biological structure and function. J Biol Chem. 2012;287(50):41576-82.

9. Blanc RS, Richard S. Regenerating muscle with arginine methylation. Transcription. 2017;8(3):1758.

10. Li WJ, He YH, Yang JJ, Hu GS, Lin YA, Ran T, et al. Profiling PRMT methylome reveals roles of hnRNPA1 arginine methylation in RNA splicing and cell growth. Nat Commun. 2021;12(1):1946.

11. Fisk JC, Read LK. Protein arginine methylation in parasitic protozoa. Eukaryot Cell. 2011;10(8):1013-22.

12. Campagnaro GD, Nay E, Plevin MJ, Cruz AK, Walrad PB. Arginine Methyltransferases as Regulators of RNA-Binding Protein Activities in Pathogenic Kinetoplastids. Front Mol Biosci. 2021;8:692668.

13. Clayton CE. Life without transcriptional control? From fly to man and back again. EMBO J. 2002;21(8):1881-8.

14. Lahav T, Sivam D, Volpin H, Ronen M, Tsigankov P, Green A, et al. Multiple levels of gene regulation mediate differentiation of the intracellular pathogen Leishmania. FASEB J. 2011;25(2):515-25.

15. Cloutier S, Laverdière M, Chou MN, Boilard N, Chow C, Papadopoulou B. Translational control through elF2alpha phosphorylation during the Leishmania differentiation process. PLoS One. 2012;7(5):e35085.

16. Tsigankov P, Gherardini PF, Helmer-Citterich M, Zilberstein D. What has proteomics taught us about Leishmania development? Parasitology. 2012;139(9):1146-57.

17. Pelletier M, Pasternack DA, Read LK. In vitro and in vivo analysis of the major type I protein arginine methyltransferase from Trypanosoma brucei. Mol Biochem Parasitol. 2005;144(2):20617.

18. Pasternack DA, Sayegh J, Clarke S, Read LK. Evolutionarily divergent type II protein arginine methyltransferase in Trypanosoma brucei. Eukaryot Cell. 2007;6(9):1665-81.

19. Fisk JC, Zurita-Lopez C, Sayegh J, Tomasello DL, Clarke SG, Read LK. TbPRMT6 is a type I protein arginine methyltransferase that contributes to cytokinesis in Trypanosoma brucei. Eukaryot Cell. 2010;9(6):866-77.

20. Fisk JC, Sayegh J, Zurita-Lopez C, Menon S, Presnyak V, Clarke SG, et al. A type III protein arginine methyltransferase from the protozoan parasite Trypanosoma brucei. J Biol Chem. 2009;284(17):11590-600.

21. Kafková L, Debler EW, Fisk JC, Jain K, Clarke SG, Read LK. The Major Protein Arginine Methyltransferase in. J Biol Chem. 2017;292(6):2089-100.

22. Kafková L, Tu C, Pazzo KL, Smith KP, Debler EW, Paul KS, et al. Trypanosoma brucei PRMT1 Is a Nucleic Acid Binding Protein with a Role in Energy Metabolism and the Starvation Stress Response. mBio. 2018;9(6).

23. Cáceres TB, Thakur A, Price OM, Ippolito N, Li J, Qu J, et al. Phe71 in Type III Trypanosomal Protein Arginine Methyltransferase 7 (TbPRMT7) Restricts the Enzyme to Monomethylation. Biochemistry. 2018;57(8):1349-59.

24. Lott K, Li J, Fisk JC, Wang H, Aletta JM, Qu J, et al. Global proteomic analysis in trypanosomes reveals unique proteins and conserved cellular processes impacted by arginine methylation. J Proteomics. 2013;91:210-25.

25. Lott K, Zhu L, Fisk JC, Tomasello DL, Read LK. Functional interplay between protein arginine methyltransferases in Trypanosoma brucei. Microbiologyopen. 2014;3(5):595-609.

26. Alvar J, Vélez ID, Bern C, Herrero M, Desjeux P, Cano J, et al. Leishmaniasis worldwide and global estimates of its incidence. PLoS One. 2012;7(5):e35671. 
27. Ferreira TR, Alves-Ferreira EV, Defina TP, Walrad P, Papadopoulou B, Cruz AK. Altered expression of an RBP-associated arginine methyltransferase 7 in Leishmania major affects parasite infection. Mol Microbiol. 2014.

28. Alcoforado Diniz J, Chaves MM, Vaselek S, Miserani Magalhães RD, Ricci-Azevedo R, de Carvalho $\mathrm{RVH}$, et al. Protein methyltransferase 7 deficiency in Leishmania major increases neutrophil associated pathology in murine model. PLoS Negl Trop Dis. 2021;15(3):e0009230.

29. Ferreira TR, Dowle AA, Parry E, Alves-Ferreira EVC, Hogg K, Kolokousi F, et al. PRMT7 regulates RNA-binding capacity and protein stability in Leishmania parasites. Nucleic Acids Res. 2020;48(10):5511-26.

30. Goyard S, Beverley SM. Blasticidin resistance: a new independent marker for stable transfection of Leishmania. Mol Biochem Parasitol. 2000;108(2):249-52.

31. Dhar S, Vemulapalli V, Patananan AN, Huang GL, Di Lorenzo A, Richard S, et al. Loss of the major Type I arginine methyltransferase PRMT1 causes substrate scavenging by other PRMTs. Sci Rep. 2013;3:1311.

32. Ruy PC, Monteiro-Teles NM, Miserani Magalhães RD, Freitas-Castro F, Dias L, Aquino Defina TP, et al. Comparative transcriptomics in Leishmania braziliensis: disclosing differential gene expression of coding and putative noncoding RNAs across developmental stages. RNA Biol. 2019;16(5):639-60.

33. Kapler GM, Coburn CM, Beverley SM. Stable transfection of the human parasite Leishmania major delineates a 30-kilobase region sufficient for extrachromosomal replication and expression. Mol Cell Biol. 1990;10(3):1084-94.

34. Doyle PS, Engel JC, Pimenta PF, da Silva PP, Dwyer DM. Leishmania donovani: long-term culture of axenic amastigotes at 37 degrees C. Exp Parasitol. 1991;73(3):326-34.

35. Beneke T, Madden R, Makin L, Valli J, Sunter J, Gluenz E. A CRISPR Cas9 high-throughput genome editing toolkit for kinetoplastids. R Soc Open Sci. 2017;4(5):170095.

36. Beattie L, Peltan A, Maroof A, Kirby A, Brown N, Coles M, et al. Dynamic imaging of experimental Leishmania donovani-induced hepatic granulomas detects Kupffer cell-restricted antigen presentation to antigen-specific CD8 T cells. PLoS Pathog. 2010;6(3):e1000805.

37. Schumann Burkard G, Jutzi P, Roditi I. Genome-wide RNAi screens in bloodstream form trypanosomes identify drug transporters. Mol Biochem Parasitol. 2011;175(1):91-4.

38. Heberle H, Meirelles GV, da Silva FR, Telles GP, Minghim R. InteractiVenn: a web-based tool for the analysis of sets through Venn diagrams. BMC Bioinformatics. 2015;16:169.

39. Daigneault M, Preston JA, Marriott HM, Whyte MK, Dockrell DH. The identification of markers of macrophage differentiation in PMA-stimulated THP-1 cells and monocyte-derived macrophages. PLoS One. 2010;5(1):e8668.

40. Gatto M, Borim PA, Wolf IR, Fukuta da Cruz T, Ferreira Mota GA, Marques Braz AM, et al. Transcriptional analysis of THP-1 cells infected with Leishmania infantum indicates no activation of the inflammasome platform. PLoS Negl Trop Dis. 2020;14(1):e0007949.

41. Späth GF, Beverley SM. A lipophosphoglycan-independent method for isolation of infective Leishmania metacyclic promastigotes by density gradient centrifugation. Exp Parasitol. 2001;99(2):97-103. 